\title{
Quand les « malheureux » deviennent des " enragés » : ethnographie de moudjahidines syriens (2012-2014)
}

When the 'desperates' become 'enraged': an ethnographic study of Syrian Mudjahidin (2012-2014)

\section{Romain Huët}

\section{(2) OpenEdition Journals}

Édition électronique

URL : http://journals.openedition.org/conflits/18948

DOI : $10.4000 /$ conflits. 18948

ISSN : $1777-5345$

Éditeur :

CECLS - Centre d'études sur les conflits - Liberté et sécurité, L'Harmattan

Édition imprimée

Date de publication : 30 juillet 2015

Pagination : $31-75$

ISBN : 979-2-343-07175-6

ISSN : $1157-996 \mathrm{X}$

Référence électronique

Romain Huët, «Quand les «malheureux » deviennent des « enragés » : ethnographie de moudjahidines syriens (2012-2014) », Cultures \& Conflits [En ligne], 97 | printemps 2015, mis en ligne le 30 juillet 2016, consulté le 07 avril 2021. URL : http://journals.openedition.org/conflits/18948 ; DOI : https://doi.org/10.4000/conflits.18948 


\section{Quand les «malheureux» deviennent des "enragés »: ethnographie de moudjahidines syriens (2012-2014)}

\section{Romain HUËT}

Romain Huët est maître de conférences en sciences de la communication (Université Rennes II). Ses travaux portent sur les rapports entre souffrance sociale et contestation. Ses publications récentes incluent: "Plongés dans l'ordinaire des combattants syriens", interview sur Médiapart (2014); "Les mots de la souffrance sociale ", Rapport de recherche, INPES/S.O.S. Amitié (2014).

Cet article revient sur les conclusions d'une enquête ethnographique d'une soixantaine de jours conduite au sein d'un groupe de combattants de l'Armée syrienne libre (ASL) à Alep et sa région (juillet 2012, janvier 2013), puis d'un groupe de moudjahidines ${ }^{1}$ affilié au Front islamique dans la région d'Hama (mai 2014, septembre 2014) 2. Littéralement intégré dans ces brigades, notre but était de décrire "l'ordinaire » de ces combattants engagés dans une lutte armée depuis plus de trois ans. La problématique générale de cette enquête est simple dans son principe : il s'agit d'approcher les ressorts subjectifs qui conduisent des individus " ordinaires ", au sens où ils ne sont a priori pas préparés à la lutte armée, à tuer et mourir à des fins politiques. En d'autres termes, il s'agit de se demander quelles sont les idées susceptibles de soutenir l'engagement dans une violence radicale. Alors que pendant de nombreuses années, ces mêmes personnes s'en sont tenues à ruminer les multiples injustices et infortunes dont ils affirment faire l'expérience depuis longtemps, ces hommes en viennent subitement à « basculer » et à donner leur vie pour les combattre, considérant ainsi, pour reprendre les termes d'Albert Camus, que « la justice est supérieure à la vie ${ }^{3}$ ».

1. Le moudjahidine désigne un combattant engagé dans le djihad.

2. Les deux premiers voyages ont été réalisés avec Soraya El Kahlaoui (sociologue, Ehess) dans la région d'Alep et les deux suivants avec Laurent l'Hermite (documentariste) à Hama.

3. Camus A., L’homme révolté, Paris, Gallimard, 1951. 
Ce terme de « basculement subjectif » désigne le processus par lequel des hommes ordinaires se transforment en des combattants. Cette question a quelque chose de fascinant car elle vise à approcher ce qui conduit soudainement ces hommes dont on ne pouvait pas toujours attendre un engagement révolutionnaire, à lever la peur de la répression et à exprimer le projet ferme d'en terminer définitivement avec ce qu'ils considéraient être une dictature.

C'est une question générale et des plus complexe. Sa complexité réside pour l'essentiel dans le fait que c'est un processus fluide, incertain et difficilement traduisible par les mots, comme l'ont déjà affirmé Jacques Sémelin ${ }^{4}$ et Laurent Bonelli ${ }^{5}$. Nombre de chercheurs ont apporté à ces questions des réponses d'ordre psychologique, politique, historique et évidemment anthropologique. L'explication est nécessairement multicausale 6 et toujours approximative. Devant la multiplicité des manières de l'aborder, le but a été de conduire une enquête ethnographique auprès des moudjahidines syriens pour tenter de comprendre empiriquement l'ordinaire de ces hommes qui, au fil du temps, sont devenus des combattants engagés dans une lutte des plus difficile car dépourvue d'horizons victorieux clairs.

Les enquêtes dans le « cours de la guerre » sont rares. Pour la plupart, elles sont réalisées dans l'après-coup du conflit, au moyen de témoignages ou par l'étude des chroniques réalisées par les combattants eux-mêmes. C'est pourquoi il sera discuté dans un premier temps de la pertinence et des limites de ce type d'enquête ethnographique dans une situation insécuritaire et peu habituelle dans le monde académique. Tout l'enjeu sera de situer les possibilités ouvertes par l'enquête ethnographique et les difficultés particulières qu'un tel travail, soumis à la pression du contexte et à l'effondrement des formes propres à l'expérience sociale de la guerre, engendre. Dans un second temps, il conviendra de qualifier sommairement ces brigades et les combattants qui la composent pour aborder ensuite la question centrale des ressorts subjectifs. Le but n'est certainement pas d'affirmer un positionnement moral (pour ou contre la rébellion). Il s'agit plutôt de s'essayer à traduire dans le langage de l'ethnographie et de la raison cette expérience d'un genre un peu particulier en tentant de comprendre comment ces hommes ordinaires, en rupture avec le pouvoir et une partie non marginale du peuple, ordonnent et justifient leurs rapports mutuels conformément à l'idée qu'ils se font du monde.

4. Sémelin J., Purifier et détruire, Usages politiques des massacres et génocides, Paris, Le Seuil, 2005 , p. 39.

5. Bonelli L., «De l'usage de la violence en politique », Cultures $\&$ Conflits, 81-82, 2011, pp. 716.

6. Browning C.-R., Des Hommes ordinaires, Paris, Texto, 2007. 


\section{Une perception géostratégique trop omniprésente : de l'intérêt de l'en- quête ethnographique}

Quatre séjours de recherche ont été réalisés en Syrie. Les deux premiers nous ont conduit dans la ville d'A'zaz au nord d'Alep (vingt-et-un jours en juillet 2012 et dix jours en décembre 2012). Les deux derniers séjours ont été réalisés à Murek à l'est de la ville de Hama, ville encore contrôlée par l'armée régulière (douze jours en avril 2014 et dix-huit jours en septembre 2014). Plusieurs raisons nous ont conduit à investir sociologiquement ce terrain.

La première est liée à un projet intellectuel plus large. Dans la lignée théorique d'Emmanuel Renault ${ }^{7}$, de Michäl Foessel 8 ou encore de Franck Fischbach ${ }^{9}$, il s'agit d'éprouver le lien entre souffrance sociale et politique. L'hypothèse est que la "révolution syrienne ", à l'instar de la plupart des luttes sociales, découle d'expériences de vie douloureuses et vécues comme injustifiées. Il s'agit donc d'aborder les luttes sociales sous le prisme de la souffrance. Cette hypothèse de départ n'est pas sans poser problème. Il a notamment été constaté que la souffrance a bien souvent des effets antipolitiques 10 en ce sens qu'une vie peut indéniablement conduire à l'impossibilité de s'approprier le monde et inciter les individus à se replier dans leur intériorité, qui devient « le seul refuge qu'il reste de ce monde inappropriable 11 ». Cependant, il est des cas où les hommes «malheureux » se transforment en des « enragés 12 ", où la haine succède à la plainte ${ }^{13}$. Le discours sur la souffrance passée constitue ainsi une ressource centrale permettant de supporter une lutte visant à transformer le monde. Cette hypothèse centrale a été testée sur le terrain d'enquête.

La seconde raison part du sentiment qu'on ne sait pas grand-chose sur les luttes armées lorsqu'elles sont en cours de réalisation ${ }^{14}$. Nous avons peu de connaissances sur le temps de guerre - temps incertain et suspendu où « la vie continue dans l'ignorance de la forme concrète que prendront les lendemains $15 »$. En anthropologie en particulier, c'est un terrain plutôt méconnu. C'est un monde étrange et étranger, si bien que l'on s'en fait souvent une idée très approximative. Ces quelques semaines sur place ont permis de ressentir l'expérience de la guerre. C'est un monde spécial ; un monde avec ses lois, ses hiérarchies, ses logiques de l'honneur, ses modes si singuliers de raisonner.

7. Renault E., Souffrances sociales, Paris, La Découverte, 2008.

8. Foessel M., Après la fin du monde, Paris, Le Seuil, 2012.

9. Fischbach F., Sans objet. Capitalisme, subjectivité et aliénation, Paris, Librairie philosophique J. Vrin, 2009.

10. Revault d'Allonnes M., L'Homme compassionnel, Paris, Le Seuil, 2008.

11. Fischbach F., Sans objet. Capitalisme, subjectivité et aliénation, op. cit.

12. Arendt H., Essai sur la révolution, Paris, Gallimard, 1967.

13. Sloterdijk P., Colère et temps, Paris, Meta-Editions, 2007.

14. Bonelli L., "De l'usage de la violence en politique », art. cit.

15. Sémelin J., Purifier et détruire, op. cit. 
Nous avions là un point d'observation directe de l'intérieur, au sein de ces deux cellules de moudjahidines, expérience qui nous paraissait contraster tant avec la littérature existante sur les luttes armées, que celle sur la Syrie, qui était le produit d'un regard lointain et qui nous semblait profondément biaisé 16 . En effet, la littérature sur la Syrie est dominée par l'approche géopolitique. Arguant de la complexité des forces en présence, opposant d'un côté l'axe de la Russie, de l'Iran, de la Chine ou encore du Liban, et de l'autre les soutiens plus lointains des pays du Golfe, des États-Unis et de l'Europe. Étrangement, en Syrie, on ne parle que des influences étrangères ${ }^{17}$. Inévitablement, elles ont existé d'abord parce que la guerre engendre des coûts. Elle nécessite des dépenses extraordinaires et des forces et des moyens considérables ${ }^{18}$. Mais les influences extérieures ne suffisent à lever une masse d'hommes qui, dès les premières heures de la révolution, s'est soulevée en étant fermement attachée à l'idée que le « devenir est supérieur à la vie 19 ». Force est de constater qu'une masse d'individus se laisse entraîner aux chances qu'offre la guerre et se socialise progressivement à la violence 20 . Toute la question est alors de savoir ce qui peut bien pousser des individus à tout sacrifier et à affronter la mort.

16. Cette justification rencontre précisément les préoccupations de Loïc Wacquant dans le travail qu'il a réalisé dans un club de boxe de la ville de Chicago : Wacquant L., "L'habitus comme objet et méthode d'investigation ", Actes de la recherche en sciences sociales, 184, 2004, pp. 108-121.

17. De manière concomitante à bon nombre de ces propos, il n'était pas rare de voir se multiplier les tentatives visant à discréditer les révolutionnaires, qui étaient immédiatement qualifiés de « rebelles » ou encore "d'insurgés » au service de nations étrangères, c'est-à-dire autant de performances linguistiques qui, pour reprendre les termes de Judith Butler, désapprouvent, délégitiment et enferment leurs expériences sociales et rendent leurs justifications incertaines (Butler J., Le Pouvoir des mots. Politique du performatif, Paris, Editions d'Amsterdam, 2004). En quelques jours, la révolution était devenue sur le plan langagier une " guerre ». Les « pro Bachar " défendaient la thèse classique de l'agression terroriste alors que les opposants parlaient d'une révolution avortée et saccagée par le pouvoir, lequel a mené une guerre contre les civils tués par les bombardements et les attaques au sol. Dès le départ, la Syrie était traversée par un mouvement dont on ne savait pas bien s'il s'agissait d'un peuple, d'un groupe ou d'une minorité qui s'était soulevé contre le régime dénoncé pour son caractère oppressif et tyrannique. Comme le souligne Ziad Majed, le glissement sémantique extraordinairement rapide de la « révolution » à la " guerre » ou à la « crise syrienne " dans les médias et les débats publics a contribué à la définition de "parties toutes responsables de la situation actuelle ». Pourtant, aux premières heures de la révolution, les manifestations de la contestation étaient des plus classiques : les slogans " dégage " fleurissaient dans la rue. Femmes et enfants se situaient en tête de cortège avec des bâtons d'oliviers en mains pour demander pacifiquement la destitution de Bachar El Assad. Quant à ce qui allait devenir leur révolution, aucun participant ne possédait la moindre prémonition du carnage qui se tramait. Voir : Majed Z., Syrie, la révolution orpheline, Paris, Actes Sud, 2014.

18. Jünger E., L'État universel suivi de La Mobilisation totale, Paris, Gallimard, 1990.

19. Comme le rappelle judicieusement Ziad Majed, les premières manifestations ont été pacifiques. Dès le départ, elles ont fait l'objet de répressions violentes par le pouvoir en place dans les villes de Damas, Deraa, Idleb, Homs, Qamichli, Amouda, Daraya ou encore Alep. Majed Z., Syrie, la révolution orpheline, op. cit., pp. 56-89.

20. C'est en ces termes qu'Albert Camus expliquait les sources du terrorisme pendant la guerre d'Algérie au moment de la représentation de sa pièce « Les Justes ». Camus A., « Les Justes », Euvres, Paris, Gallimard, 2013, pp. 781-843. 
L'enquête ethnographique trouve ici sa pleine justification en une réaction critique à la lecture strictement géopolitique du conflit. S'il ne s'agit pas de contester la pertinence de telles approches, force est de constater qu'elles ont pour effet d'étouffer et de rendre les voix des insurgés inaudibles ou confuses. La conséquence première de cette invisibilisation des motifs profonds qui animent les combattants est d'hypothéquer leurs voix et de rendre les justifications sociales incertaines. D'ailleurs, à voir comment les combattants nous ont parlé de la légitimité de leur combat des heures durant, on comprend bien que cette volonté de se rendre audible est une «lutte pour la vie, contre l'effacement, contre la disparition $21 »$. En somme, comprendre ce qui anime les révolutionnaires pour qu'ils osent payer de leur vie est une question élémentaire à se poser devant une telle situation. C'est une manière de faire droit aux signes de vie qui demeurent dans ce monde muet, où le temps donne toujours l'impression de ne pas s'écouler. C'est de ces logiques et de ces rationalités propres aux combattants qu'il convient de débattre.

Formulée ainsi, la question de départ porte une ambition irréalisable. L'ethnographie n'est pas en mesure de répondre à un problème aussi vaste et complexe. Dans notre cas de figure, cela est d'autant plus vrai. L'enquête est confinée à un cadre réduit. Pour reprendre les termes d'Aimé-Jules Bizimana ${ }^{22}$, c'est une " enquête de proximité » car elle n'est effectuée qu'au sein de deux brigades de moudjahidines dans une durée relativement limitée (soixante et un jours). Ainsi, le chercheur en quête de certitudes est nécessairement débordé. Cette enquête "vue d'en bas ", au sens où elle décrit le quotidien de quelques combattants et le rapport subjectif qu'ils entretiennent à leur lutte, ne peut prétendre à une vue globale de la guerre en Syrie. Elle n'est qu'une vision étroite et isolée d'un ensemble bien plus ample qui peut difficilement se passer d'un éclairage historique, politique et culturel. Par exemple, cette enquête n'apporte aucun éclairage significatif quant à la stratégie de guerre ou à la coordination entre les cellules de combattants ou encore aux liens qu'ils entretiennent avec des mécènes ou des soutiens étrangers. En bref, c'est une enquête à « ras le sol » dont l'intérêt principal est de rendre compte de l'ordinaire de ces hommes, des significations qu'ils donnent à leur lutte et de leur manière d'aménager leurs rapports mutuels et leur existence au jour le jour dans les ruines de la guerre. C'est là que cette sociologie de l'ordinaire trouve sa pleine justification, dans sa capacité à envisager la guerre à hauteur d'hommes. Évidemment, il est un lieu commun que de reconnaître que bon nombre de processus d'ensemble ont favorisé l'expérience sociale de la révolution puis de la guerre. Ces facteurs historiques, sociaux et politiques ne sont pas ignorés. Seulement, le point de vue développé dans cet article est strictement ethnographique au sens où les conclusions ne sont issues que des obser-

21. Le Blanc G., L'invisibilité sociale, Paris, PUF, 2009.

22. Bizimana A.-J., Le Dispositif embedding. Surveillance et intégration des journalistes en Irak, Québec, Presses de l’Université du Québec, 2014. 
vations réalisées et du sens attribué par les combattants à leur entrée progressive dans la lutte armée. Ces cellules de combattants ne sont pas nécessairement représentatives d'un ensemble. Elles n'en demeurent pas moins utiles car elles sont des fenêtres sur l'ordinaire de la guerre et de ses combattants. C'est ainsi qu'elles ouvrent la possibilité de mettre en scène un ensemble de questionnements plus que de certitudes théoriques péremptoires. L'analyse porte ainsi sur la confrontation des observations et des témoignages. Le lecteur intéressé trouvera en particulier dans l'ouvrage de Ziad Majed 23 un précieux travail récent de contextualisation historique, politique et sociale de la situation syrienne.

\section{Une immersion au cœur de deux groupes de moudjahidines : les condi- tions concrètes de réalisation de l'enquête}

Pour saisir la manière dont les combattants aménagent leur existence en rapport à l'idée qu'ils se font de leur engagement et du monde, nous avons rejoint deux cellules de moudjahidines au cours de quatre séjours. La première était affiliée à l'ASL au nord d'Alep (juillet et décembre 2012), alors que la seconde appartenait au Front islamique ${ }^{24}$ (avril et septembre 2014) et se trouvait dans la ville de Murek, ville de front partagée avec l'armée régulière de Bachar El Assad.

\section{Des cellules de combattants sociologiquement comparables}

Ces deux cellules ont de nombreux points communs. Composées d'environ cent cinquante hommes ${ }^{25}$, elles se sont constituées davantage sur une base territoriale que sur un programme idéologique précis. Aucun des hommes rencontrés (une soixantaine d'entretiens ont été réalisés) n'appartenait au préalable à un parti politique, à un mouvement associatif ou à un courant idéologiquement déterminé. Leur passé militant est faible voire inexistant. Bien sûr, tous ont connu de près ou de loin les bains de sang de 1982 dans la ville de Hama, événement qui est aujourd'hui utilisé comme une référence historique constante de la part des combattants. Tous ont donc vécu une histoire traumatique antérieure.

Ces hommes partagent la caractéristique d'être « combattants ordinaires » au sens où $80 \%$ d'entre eux n'étaient pas des militaires de carrière. Ces deux brigades ont donc en commun d'être composées d'hommes sans expérience

23. Majed Z., Syrie, la révolution orpheline, op. cit.

24. Le Front islamique a été créé le 23 novembre 2013 suite à une fusion entre le Front islamique de libération syrien (islamistes dit « modérés ») et le Front islamique syrien (salafiste). Il s'agit d'un des plus importants groupes « rebelles » en Syrie devant l'ASL, l’État islamique en Irak et au Levant et le Front Al-Nosra.

25. Nous n'avons rencontré aucune femme combattante. Si nous avons réalisé trois entretiens avec des femmes liées à la résistance, la quasi-totalité de l'enquête repose sur le témoignage de combattants masculins. 
aucune des combats, à l'exception des plus âgés qui ont vécu le soulèvement avorté d'Hama. Pour l'essentiel, il s'agit donc de civils qui ont progressivement rejoint ces brigades. Généralement, ils ont adhéré à celle la plus proche de leur maison ; brigade qu'ils ont pu connaitre par la réputation locale au moyen du bouche-à-oreille. Les moudjahidines sont donc des "gens du coin ». Dans la quasi-totalité des cas, quand ils n'ont pas de liens de parenté, voire de filiation, ils se connaissent au moins de nom. Ceci explique la forte cohésion qui parait exister entre les uns et les autres. Ancrés depuis longtemps sur leur territoire, les moudjahidines, tous sunnites, sont fortement unis et se connaissent depuis plusieurs générations. Avant la « révolution », ces hommes exerçaient des professions modestes : chauffeurs routiers, commerçants, agriculteurs, avec en guise d'appoint des " petits boulots " parallèles à ces emplois. Pour la majorité d'entre eux, ils proviennent donc des couches populaires de la société syrienne. D'après leurs mots, ils ne jouissaient pas d'un niveau de vie économique conforme à leurs aspirations. 20 \% d'entre eux étaient autrefois des militaires de carrière qui ont déserté l'armée régulière. Si la majorité des combattants ont une profession, une dizaine sont étudiants, en sciences dures, en géographie ou encore en communication. De ce fait, la majorité des combattants est jeune, avec une moyenne oscillant entre 20 et 30 ans, avec une présence rare des plus de 50 ans. Les chefs de ces brigades, comme cela semble être le cas un peu partout, sont soit des notables locaux, soit des anciens militaires de carrière. Les premiers tirent principalement leur légitimité des investissements financiers auxquels ils ont pu consentir au moment où la révolution s'armait et de l'autorité (coutumière, religieuse, administrative) dont ils jouissaient dans le passé. C'est ainsi qu'ils ont constitué les premières brigades en réunissant et en armant quelques hommes dont ils étaient proches. Ils tirent également leur légitimité des nombreux contacts à l'étranger qu'ils ont pu nouer dans le cadre de leurs activités professionnelles antérieures. Les seconds, quant à eux, sont reconnus pour leurs compétences particulières dans l'expérience de la guerre et dans le commandement des hommes.

Hétéroclite et disparate, cette population de combattants partage une référence constante à l'islam. À ce titre, on pourrait penser qu'il existe un profond clivage entre l'ASL et le Front islamique. C'est vraisemblablement le cas aujourd'hui alors qu'au départ, ce clivage n'existait pas ou ne se ressentait pas dans les justifications des combattants. En 2014, l'ASL est vivement critiquée pour son allégeance aux pays occidentaux et pour son incapacité à endiguer la corruption et le désordre dans les territoires qu'elle contrôle. C'est ainsi que depuis 2013, une large partie des combattants a quitté les rangs de l'ASL soit pour fuir la guerre, soit pour rejoindre d'autres brigades moins critiquées dans le «monde des combattants». Sur ce point, le Front islamique jouit d'une excellente réputation. Là encore, la question idéologique n’est pas décisive dans le choix des combattants de rejoindre le Front islamique. Plusieurs critères sont cruciaux et priment sur le projet intellectuel et politique que porte l'organisation en question : 
- L'activité de la brigade : puisque bon nombre de brigades s'arrêtent de combattre une fois qu'un territoire est libéré, les combattants ont tendance à la quitter pour une autre toujours "en activité ", c'est-à-dire aux premières loges sur les lignes de front. Dans les ordres de grandeur de la guerre, la présence au front est la plus importante des valeurs des combattants.

- Les conditions matérielles de vie à l'intérieur de la brigade : cela concerne tout aussi bien l'équipement militaire que la solde accordée aux combattants (une moyenne de 75 dollars par mois), la capacité de la brigade à prendre en charge financièrement et socialement les familles des martyrs. Pour les combattants, ce type d'organisation est le signe d'une certaine droiture dans l'organisation de la vie de la brigade.

- La présence de personnes connues au sein de ces brigades (familles et ou amis).

En moyenne, les combattants ont changé deux à trois fois de brigade pour ces trois raisons.

Nous reviendrons sur la question religieuse. Ici, il importe de souligner le fait que les combattants ne rejoignent pas des brigades en fonction d'un projet politique particulier ou d'une idéologie précise mais pour des raisons plus pratiques dans la vie courante de la guerre.

\section{Dispositif méthodologique : une immersion concrète au sein des brigades}

Concrètement, au niveau de la méthodologie, cinquante-six entretiens ont été réalisés avec des combattants. De manière tout à fait classique, ces entretiens avaient pour but de les inciter à opérer un retour sur leur propre engagement dans le mouvement de contestation et sur la radicalisation qui s'en est suivie. Dans ces entretiens, toute la question était de savoir comment ces gens ordinaires étaient devenus, au fil du temps, des combattants. Chacun nous racontait son histoire, ses troubles, ses souvenirs pénibles et son exaspération qui ont conduit à leur indignation puis à leur engagement armé. Il nous a d'ailleurs semblé que, d'une manière générale, ils n'aimaient pas la raconter. Quand nous sollicitions ces souvenirs au cours de nos entretiens, c'était manifestement pour eux un exercice pénible et délicat, et ils s'efforçaient visiblement à ne pas trop penser au passé. Ils ne racontaient pas leurs histoires personnelles de façon spontanée.

Beaucoup avaient une manière très particulière de la raconter : raide, détachée et extérieure. Lorsque nous les invitions, ils racontaient avec une précision inégale leurs souvenirs effrayants et étranges. Certains semblaient déjà effacés alors que d'autres se confondaient, ne laissant ainsi qu'une impression 
d'ensemble dépourvue d'une chronologie précise et stable. C'est ainsi, que nous avons pu recueillir de nombreux récits qui témoignaient de leur perplexité, de leur impatience, de leur colère, de tous ces questionnements tourmentés d'eux-mêmes. Récits qu'il a fallu croiser pour en repérer les régularités quant à la manière de mettre en sens leur engagement.

Du point de vue de l'observation, nous avons suivi plusieurs combattants dans leurs activités ordinaires. En temps de guerre, il existe aussi un quotidien fait de régularités. En effet, les longues journées sont occupées par les combats, l'attente entre deux fronts et les tâches domestiques nécessaires au bon fonctionnement de la brigade. Tous se donnent de la peine à accomplir ces diverses tâches selon une répartition minutieuse. Il y a aussi les longues heures d'ennui au cours desquelles les combattants attendent les nouvelles missions. C'est au cours de ces moments qu'ils bavardent, échangent des ragots ou s'informent.

Généralement, et principalement dans la ville de Murek, nous sommes restés avec quatre d'entre eux. Par écrit, nous notions les situations dont nous étions témoins, nous enregistrions par dictaphone et parfois par vidéos les scènes, les différents temps de la journée et les comportements. Nous avons partagé leur repas, leurs nuits, nous avons veillé ensemble, prié ensemble pensant voir la mort venir durant les bombardements. Nous avons manifesté en leur compagnie, assisté à des réunions de coordination, participé à des obsèques. Ils nous ont conté leurs manières d'administrer les territoires libérés, leurs façons d'organiser les secours et de se coordonner avec d'autres cellules. Nous avons rendu visite à certains d'entre eux dans ces hôpitaux de fortune où se concentrent les horreurs de la guerre, les gémissements et les cris de douleur des blessés. Dans bon nombre de situations, nous nous sommes tenu en retrait, en silence, afin d'approcher le monde ambiant de leur quotidienneté, de filmer ou d'enregistrer par dictaphone ou encore de prendre en notes les conversations qu'ils tissaient entre eux dans les moments de repos. Nous les avons également accompagnés sur les lignes de front, réalisant ainsi les observations et les entretiens dans le vacarme des armes, en présence de la destruction et au milieu de la sombre douleur.

Le choix de rejoindre les lignes de front est réfléchi. Elles ne sont pas de mauvais endroits pour réaliser nos observations. D'abord parce qu'il s'agit de « leur » quotidien. Ensuite, dans le creux des bombardements, les lignes de front sont aussi des endroits tranquilles où se tiennent de nombreuses séances de bavardage en commun bien moins régulées qu'à l'accoutumée. Elles révèlent des tranches de vies simples, sans ambages. Autour d'un thé, nous observions des combattants qui bavardaient en toute tranquillité ; ils s'adonnaient à quelques conversations légères et familières, ils parlaient des journées paisibles d'autrefois, ils débattaient plaisamment de leurs expériences communes, ils 
discutaient de l'allure des combats, ils s'imaginaient les vies telles qu'elles se déroulent dans les pays en paix, ils se donnaient des nouvelles du monde comme s'il s'agissait d'échapper au "ver rongeur des pensées » selon les termes d'Ernst Jünger. Il n'était d'ailleurs pas rare de voir le front s'égayer et mettre en vie ces visages fatigués et creusés au cours du déballage de ragots racontés avec un sens évident de la taquinerie, comme en témoigne cette conversation sur le front entre trois combattants, Abou Mossad, Abou Muhammed et Abou Ryan :

Abou Mossad, un combattant à la carrure imposante arrive essoufflé auprès de la brigade, à quelques mètres de la ligne de front : « les gars, les gars, je l'ai échappé belle. J'étais à quelques mètres de gars de l'armée régulière. Je leur ai tiré une roquette mais elle n'a pas explosé ! Ils m'ont vu, j'ai dû fuir en courant, je vous jure, je l'ai échappé belle ! »

Abou Muhammed : «non, ne jure pas !! »

Abou Mossad : «si je te jure, ils étaient à quelques mètres de moi. » Abou Ryan : " ah ça c'est pas possible, y'a que pour manger que tu cours. » [rires]

(Extrait d'une conversation entre les combattants)

Quelques minutes plus tard, les talkies-walkies annoncent l'arrivée imminente d'un jet et de deux hélicoptères sur notre zone. De l'aéroport d'Hama, il leur faut cinq minutes pour se trouver sur notre zone. Les combattants ne sourcillent pas. Ils se font quelques politesses autour du thé, servi au coin de la rue :

Talkie-walkie : « un jet et deux hélicoptères, à Murek, un jet et deux hélicoptères à Murek...»

Les combattants en cœur : «Unité de Jabha! » (crié cinq fois)

Abou Mossad : "Ils sont où là les gars de l'armée régulière ?»

Abou Ryan : "Ils sont là-bas, à cinquante mètres, même moins de cinquante mètres. Tu vois la pierre là-bas, et ben c'est là, même pas cinquante mètres. Il y aura pas mal de cartouches là [des tirs pendant les bombardements], donc si tu restes là, c'est bon. »

Talkie-walkie : «Le jet et les deux hélicoptères sont en direction de Murek (répété deux fois), que Dieu nous protège. »

Abou Muhammed: "quelqu'un veut du thé ? Tenez, buvez du thé, donnez-moi vos verres. » [Les combattants n'ont qu'un verre pour deux]

Abou Mossad : «prends ce verre, je vais boire avec lui. » [Abou Ryan]

Abou Muhammed : «non, non, tiens prends ce verre. [Abou Muhammed tend un verre de thé à Abou Hassan] T’en veux ? » 
Abou Hassan : « non vas-y, t'en as pas pris toi. »

Abou Muhammed : «non t'inquiète, c'est pas grave. »

Abou Hassan : «Non il est à toi celui-là. »

Abou Muhammed: «je te jure que c'est toi qui le prends. »

Abou Hassan : «non ne jure pas... merci. » [il prend le thé]

Ces conversations anecdotiques témoignent de la multitude de moments relâchés qui se joue au quotidien. Elles nous montrent ce que vivre signifie en de telles situations de désolation et de destruction. Ces temps sont précieux pour collecter et répertorier ces réalités les plus concrètes et les ordres normatifs qui prévalent au sein de ce «monde des combattants». Autrement dit, dans l'intermède des bombardements et des sourdes explosions, il est possible d'entrevoir ce quotidien qui ordonne le rapport à la vie, les soutiens mutuels, l'organisation concrète des brigades, la teneur des rapports mutuels et les configurations psychologiques nécessaires pour faire face à ce type de situation. Les suivre en ces endroits est une manière de s'ouvrir à leur monde et d'approcher plus concrètement le sens de leurs discours en les rapprochant de leurs expériences vécues. C'est également une manière de constater comment les hommes, dans les ruines de la ville et le désastre subjectif qu'une guerre engendre, bâtissent et aménagent leur existence pour y mener une vie supportable avec de légers moments de décontraction, faits de plaisanteries, de jeux, de chants ou de simple temps de repos.

Enfin, le fait même de rejoindre ces lieux tend à briser les distances humaines, particulièrement dans les moments de dangers. À plusieurs reprises, alors que des avions ou des hélicoptères tournoyaient au dessus de nos têtes, faisant vibrer patiemment l'idée de la mort, nous avons été en proie à la même condition que les combattants, pour le dire vite, écrasés par le sentiment redoutable de l'impuissance où le corps attend son destin ${ }^{26}$. Dans ces situations, il n'est pas exagéré de dire que l'on partageait la même condition. Leurs habitudes, leur espèce de tranquillité insouciante, leur fatalisme devenaient aussi les nôtres, du moins dans la forme et au cours de ces situations provisoires, puisque sur le fond et en général notre situation était fondamentalement différente. D’ailleurs, plusieurs nous disaient : "vous êtes des hommes comme nous, vous risquez vos vies alors même que vous êtes loin de chez vous. » Quand on nous introduisait auprès de nouveaux interlocuteurs, nos «passeurs » expliquaient que nous restions sur le front et que, comme tout un chacun ici, nous vivions les bombardements, racontant ainsi les multiples péripéties dont nous avons pu faire l'expérience. Le partage de ces situations critiques crée de la proximité. Il tisse des liens et renforce la confiance, et d'une certaine manière, il rend réelle notre présence en ce milieu ${ }^{27}$.

26. Les cellules que nous avons investies n'ont aucun équipement antiaérien. Dans ces deux brigades, nous n'y avons trouvé que quelques rares vieilles Doushkas, des mitrailleuses lourdes anti-aériennes.

27. Geertz C., «Jeu d'enfer », Le Débat, 7, 1980, pp. 86-146. 
Bien sûr, les lignes de front ne constituent pas l'unique lieu de vie des combattants. Au sein de leurs quartiers généraux (QG), chacun reprend son souffle entre deux combats. À l'intérieur de ces anciennes maisons de civils réaménagées pour l'occasion et à quelques kilomètres des lignes de front (entre cinq et dix kilomètres), la vie peut être tranquille et plus régulière. Dans les QG, les temps annulés où rien ne se passe vraiment sont nombreux. Généralement, les combattants y restent $48 \mathrm{~h}$ lors de leur véritable repos ${ }^{28}$, et quelques heures seulement lorsqu'ils sont en charge de combattre. Au cours de ces temps, les combattants se relâchent. Rivés sur leurs portables, ils jouent à des jeux vidéo, principalement des jeux de guerre, mais parfois aussi des jeux plus récréatifs comme Candy Crush ou des jeux de football. La présence massive de ces distractions en ces lieux à quelque chose d'étonnant. C'est comme s'ils réintroduisaient des situations finalisées dans ces temps d'attentes. Dans le contexte d'un présent effondré, le jeu calme les angoisses et comble le vide d'un temps profondément déchiré et désordonné. Ils écoutent aussi de la musique, dorment et s'adonnent à des soirées de bavardage. En ces temps-là, l'ordinaire paraît parfois déconnecté du désastre de la situation. Ces heures de camaraderies ou de simples repos nous éloignent étrangement de la guerre. Néanmoins, cette apparente tranquillité est toujours menacée, particulièrement par les bombardements fréquents. Lors de nos deux derniers séjours, nous avons enregistré pas moins de vingt à trente bombardements quotidiens sur notre zone. L'inquiétude tournoie sans repos dans l'esprit des combattants. À la moindre alerte, tout le monde s'agite, bouillonnant et prêt à agir pour tirer vainement avec leur kalachnikov sur les avions ou les hélicoptères ou tout simplement pour guetter le baril 29 chuter et se protéger. Ainsi, le combattant se construit toujours dans cette alternance entre la vie lente et l'inquiétude ambiante née du vacarme de la guerre. Il y a évidemment beaucoup à raconter de l'ordinaire des combattants. L'ordre existe, sans aucun doute. Mais c'est précisément l'incertain qui domine et parfois, l'indescriptible, les ruptures et les changements qui se réalisent toujours en des temps extrêmement contractés. D'une seconde à l'autre, la lenteur peut être interrompue et tout peut changer radicalement.

La description du dispositif méthodologique indique que l'enquête ethnographique est formellement des plus classiques. Elle est considérée ici comme un langage, une manière d'évoquer le monde, une façon d'organiser un contact singulier avec le réel sur la base d'observations empiriques et d'entretiens avec les individus concernés par la lutte. À l'instar de n'importe quelle autre, elle se donne pour tâche d'offrir une accessibilité partielle au monde. Néanmoins, le contexte d'une situation critique, voire extrême, altère de

28. Sur le front, les combattants réalisent des rotations de $24 \mathrm{~h}, 28 \mathrm{~h}$ ou $72 \mathrm{~h}$.

29. Les barils sont remplis de divers explosifs, de débris de ferraille, parfois d'engrais chimiques et de combustibles. Les barils sont largués à la main, préalablement allumés et poussés hors d'un hélicoptère (deux barils par hélicoptère). Au cours de nos deux derniers séjours, une trentaine de barils explosaient chaque jour sur la zone. 
manière considérable les possibilités de saisir de manière scientifique ce monde 30 . Cette enquête est insuffisante, fragile et, à de multiples égards «scientifiquement impure ». Elle n'indique aucune conclusion mais seulement des suggestions. Les ébauches d'explication, quand elles existent, sont vagues et tout aussi partielles, puisqu'elles portent sur deux cellules modestes de moudjahidines. Plusieurs difficultés altèrent le principe même de « l'esprit ethnographique 31 ». Nous en discuterons ici quelques-unes.

\section{Quand on ne fait "qu'effleurer un monde » : enquêter dans un contexte chaotique}

Comme tout terrain d'enquête, l'étude de l'ordinaire est toujours « irrésolu et problématique 32 ". Dans ce cas précis, son caractère problématique est peut-être encore plus manifeste. C'est un compte rendu réservé et nécessairement prudent. En premier lieu, et c'est là une première conclusion de notre enquête ethnographique, il nous faut reconnaître que nous n'avons fait qu'effleurer leur "monde ». Nous n'avons qu'à peine entrevu leur système de valeurs ou encore l'organisation concrète de leur existence. En contexte de conflit armé, il est difficile de rendre compte d'un ordinaire. Quand nous relatons des anecdotes ou des histoires singulières, nous ignorons souvent si elles sont représentatives d'un ensemble. Difficile également de figurer des ordres de grandeur. Pour exemple, dans la seule région d'Hama, nous n'avions aucune idée de la masse des groupes effectivement actifs et engagés dans la lutte. Faute d'éléments empiriques, l'argumentation n'est que très rarement soumise à la preuve chiffrée. Notre séjour de soixante-et-un jours est bien trop bref. Il ne peut saisir que des situations fugitives, instantanées, presque versatiles. Le risque d'échouer est vif en ce point précis que l'on risque l'empressement du discours journalistique qui seulement "se hâte à maîtriser le présent sans avoir compris le passé 33 », mais plus encore, nous risquons de nous contenter de produire un compte rendu fragmentaire et photographique sans les rapporter à « des configurations de relations sociales des milieux de ressources et de contraintes 34 ». Le danger de ce type d'enquête en conflit armé est d'arracher en vitesse quelques expériences ordinaires, saisies sur le vif, quelques témoignages partiels et de repartir aussitôt avec ce maigre butin. C'est l'effet que produisent en nous ces quatre séjours. La connaissance qui est susceptible d'en être retirée est nécessairement suspecte. Il est difficile d'en extraire quelques catégories conceptuelles isolées de leur contexte sans manifester quelques impuretés et imprécisions de la pensée.

30. Amiraux V., Cefaï D., « Les risques du métier. Engagements problématiques en sciences sociales ", Cultures E Conflits, 47, automne, 2002, pp. 15-48.

31. Cefaï D. (ed.), L’Engagement ethnographique, Paris, Éditions de l'EHESS, 2010.

32. Chauvier X., Anthropologie de l'ordinaire, une conversion du regard, Toulouse, Anacharsis, 2011, p. 80.

33. Benjamin W., Euvres II, Paris, Gallimard, 2000.

34. Cefaï D., «Faire du terrain à Chicago dans les années cinquante », Genèses, 46, 2002, pp. 122137. 
C'est la raison pour laquelle, plutôt que de forcer l'ordre et le sens, peutêtre est-il utile dans ce contexte de livrer ces situations précaires et étranges à la réflexion scientifique. On sait bien que l'exigence de l'écriture ethnographique implique une certaine rigueur. Dans ce cas présent, elle a pour visée d'accueillir le vague et le confus. Les explications que nous apportons se hasardent à perpétuer et à reconduire l'incomplétude, l'obscur, voire l'inexplicable. C'est comme s'il s'agissait d'avancer sur ces chemins qu'on encombre d'explications incomplètes et maladroites à mesure qu'on les dégage. La restitution de l'enquête ethnographique veut produire cette impression de maladresse et de désarroi plutôt que de forcer la mise en ordre. On ne peut s'en tenir à ce qui compte; on ne peut atteindre la vérité de ces basculements subjectifs qui poussent des hommes à soudainement accepter de tuer et de mourir. En conséquence, les descriptions font ressentir plus qu'elles ne donnent à voir. Elles ne distinguent que trop superficiellement des attitudes typiques et régulières. Elles font ressentir un monde d'hommes et de choses globalement inorganisé, ou un ordre tellement précaire que sa vocation paraît être celle de la désorganisation à venir.

Cette confusion s'explique essentiellement par le fait que dans ce terrain, se joue un effondrement des formes au bénéfice de quelque chose de précaire, d'aménagements provisoires, d'une instabilité générale qui menace constamment les choses. La configuration même des paysages en est l'exemple typique. À A'zaz, Alep ou Murek, le paysage de poussière, d'acier et de gasoil est toujours insaisissable. Il est fait d'irrégularités et de changements liés au déplacement incessant des lignes de front et aux destructions permanentes. À mesure que nous nous avançons dans ces villes désertes ou que nous nous enfonçons vers les fronts étroits, au milieu des décombres, des murs éventrés et des rues dévastées, il est difficile d'accrocher ses yeux à des points fixes. L'ouie est particulièrement affectée. Il faut s'accommoder au vacarme des tirs de Kalachnikov, aux sourdes explosions, au bourdonnement lointain et régulier des avions et des hélicoptères qui signale l'approche imminente de la violence et de la menace. La crainte fait naître une attention pour les moindres détails. Les nerfs sont tellement à vifs que nous vivions de «nombreuses illusions auditives, en vertu desquelles le roulement de chaque voiture devenait le ronflement de mauvais augure de l'obus meurtrier 35 ». Chaque bruit est décortiqué et mal interprété. Enfin, il y a le moment du danger imminent, lorsque ces mêmes engins tournoient au dessus de nos têtes à intervalles réguliers. En ces moments, les voix se taisent. C'est un silence lourd comme si l'espace s'était vidé de son air. C'est un silence qui précède le vacarme et l'agitation générale. De toute évidence, ces moudjahidines vivent un temps annulé où, dans le hurlement sourd des bombardements et les sifflements des barils qui paraissent déchirer le ciel, ils entendent l'appel de la tombe. Puis, les barils s'écrasent. Des colonnes de fumées épaisses s'élèvent et colorent le ciel, ajou- 
tant au paysage désolé ce navrant mélange de violence, de misère matérielle, de désorientation et de triste précarité. Régulièrement, en tant qu'enquêteur, nous sommes inquiet, nerveux, agité, mal à notre aise. Il est inévitable que la faculté de penser «dans le cours de l'observation » soit souvent paralysée et que les impressions connaissent ici ou là quelques débordements.

En somme, c'est un paysage oppressé, bruyant et sombre. Un paysage qui produit ce sentiment indicible de désolation et cette étonnante sensation de ne pas appartenir a ce que l'on voit. Entouré par les ruines, il est difficile de repérer ce que l'apparence de chaos cache comme configuration. Car la guerre a sa régularité et ses ordres. Il y a des habitudes ; les journées de combats qui commencent au lever du soleil et qui se calment à l'arrivée de la nuit, ces longs temps d'attentes qui inspirent cette sévère monotonie. Surtout, il y a les conversations favorites des combattants, les services indispensables pour organiser et faire fonctionner le quotidien, et bien d'autres tâches ou situations régulières. Seulement, l'omniprésence des ruines altère le regard soucieux de l'observateur dans la mesure où elle produit une crise radicale du rapport signifiant au réel ${ }^{36}$. Il y a quelque chose d'invraisemblable et de démesuré. Ce paysage mortifère laisse étourdi et hagard. L'enquêteur est face à un contexte étouffant et hanté par cette vision épouvantable d'un monde livré à la mort. En définitive, c'est comme si le monde se soustrayait à la narration pour ne se livrer qu'en affects.

Par ces longues descriptions, nous cherchons à imaginer ce qu'un regard ethnographique est susceptible de déceler dans le cadre d'un paysage apocalyptique et dans un contexte étouffant d'irréalité. Souvent, en tant qu'enquêteur, nous avons éprouvé ce sentiment décourageant de ne rien comprendre, c'est-à-dire d'observer ces hommes s'agiter obstinément sans comprendre précisément ce qu'ils font. De toute évidence, il a été difficile de se former une idée claire de ce quotidien car les manifestations subjectives des combattants sont étranges, parfois confuses et contradictoires. C'est un regard qui traque l'ordre de ces discours et de ces actes dans le creux des décombres, des paysages dévastés, et des attaques régulières de l'armée de Bachar El Assad. Indéniablement, de telles circonstances altèrent l'esprit. Nous ressentons cette vive impuissance à dominer intellectuellement le sujet, à saisir cette guerre tant dans son devenir que dans son orientation. Notre impression est que nous ne voyions que les fragments et jamais les racines des choses, même si, au fur et à mesure de nos séjours, nous avons une vue plus nette qu'auparavant.

\section{Composer avec les aspérités du réel}

À cette désorientation liée aux paysages dissonants s'ajoutent les contraintes concrètes liées au contexte insécuritaire. Toute liberté de mouve-

36. Foessel M., Après la fin du monde, op. cit. 
ment est empêchée. Il n’y a ni refuges, ni bases totalement protégés. L'engagement dans une telle recherche est éprouvant physiquement : quelques heures de sommeil, l'humidité et le froid durant l'hiver, des conditions sanitaires critiques, en plus de l'évidente confrontation physique aux horreurs de la guerre. Un tel terrain requiert aussi des forces psychiques. Étonnamment, et comme le soulignait Vincent Romani dans le contexte palestinien, c'est le retour à la routine universitaire qui est le plus complexe : "La réadaptation à la vie sociale et académique policée, la distanciation vis-à-vis d'une expérience existentielle peuvent s'avérer plus difficiles que l'enquête même ${ }^{37}$. » Il ne s'agit pas tant de faits traumatiques qui posent problème que l'habitude d'une " situation permanente de dépossession de soi » et d'une intensité qui rompt avec le flux lent, progressif et régulier des routines académiques. Et en même temps, il faut bien reconnaitre que ce terrain a quelque chose de saisissant sur le plan subjectif. Il y a chez l'enquêteur un certain enchantement pour la guerre. D'ailleurs, le danger existe assurément d'entrer dans un exotisme guerrier qui, selon les termes de Walter Benjamin ${ }^{38}$, « sous le prisme d'une exaltation juvénile, déboucherait sur une glorification de la guerre dont le chercheur dépaysé se ferait l'apôtre ». L'exotisme n'est pas un sentiment superficiel. Ce terrain procure l'enivrement d'une aventure incertaine. Il y a cette «ivresse » si bien décrite par Ernst Jünger ${ }^{39}$, à rejoindre des hommes qui donnent leur vie pour une cause qu'ils considèrent juste. En quelque sorte, il y a cette excitation du péril et de l'inhabituel. La guerre produit toute une série d'expériences nouvelles dont certaines ne manquent pas d'exciter. La plus déterminante est sans doute celle de se retrouver auprès de ces hommes dont l'action détermine l'avenir. À cela, il faut sans doute ajouter la curiosité malsaine de l'expérience des bombardements, de ses manifestations et de ses effets. Enfin, probablement, il y a cette fierté de prendre courage et d'accepter de faire l'expérience de ces situations critiques au péril de sa propre vie. Bref, ce sont toutes ces impulsions fondamentalement humaines et émotionnelles qui pourraient bien étouffer nos erreurs factuelles et nos nombreuses approximations. Ce danger de l'exotisme est voué à être noyé dans la perspective glaciale du raisonnement ethnographique pour éviter précisément l'exaltation juvénile décrite plus haut et qui n'apporterait pas grand-chose aux connaissances sur les luttes armées actuelles.

Pour terminer, il y a évidemment le positionnement de l'enquêteur quelque peu ébranlé par le spectacle qu'il voit. L'exercice du discernement et de la distance n'est pas aisé. En ce contexte aussi insécuritaire que celui de la Syrie, l'empathie avec les combattants est non seulement indispensable, mais elle est surtout normale. Théoriquement, « l'empathie » n'est pas problématique en ce que le but est de les comprendre et non de les juger moralement,

37. Romani V., «Enquêter dans les Territoires palestiniens ", Revue française de science politique, 57/1, 2007, pp. 27-45.

38. Benjamin W., Euvres II, op. cit.

39. Jünger E., La Guerre comme expérience intérieure, Paris, Christian Bourgois, 1997. 
en les approuvant ou en les désapprouvant. Évidemment, pour les comprendre, ne serait-ce qu'un peu, il a fallu en faire preuve. Seulement, cette empathie a ici quelque chose d'assez radical. Elle est notamment vivement accentuée par le contexte insécuritaire qui nous fait dépendre intégralement de nos enquêtés pour nos vies; ils sont en charge de nous protéger des enlèvements ou lors des passages des check points. Lors des bombardements ou au cours de nos circulations sur les lignes de front, ils nous instruisent sur les attitudes élémentaires à adopter. En bref, les circonstances conduisent tout entier l'enquêteur à faire "profil bas » et à être plus enclin à témoigner une bienveillance radicale à l'égard de ces hommes qui prennent soin de lui. D'ailleurs, dès le départ, il est assez clair que notre premier contact a été amical et sympathique. Nous témoignons d'un regard de soutien avec les yeux de l'amitié humaine. Nous échangions sur nos vies réciproques, vivant plusieurs jours en promiscuité totale dans des conditions parfois extrêmement pénibles. Notre adhésion, notre soutien et notre aide matérielle à la lutte ont été commandés par les nécessités des circonstances. Mais plus encore, il y a des situations qui brisent les distances humaines, particulièrement dans les moments de dangers. Lorsque, à plusieurs reprises, alors que des avions ou des hélicoptères tournoyaient au dessus de nos têtes, nous nous sommes retrouvés allongés à terre dans des champs inconnus ou sous des pistachiers, inutiles comme seuls abris, il est évident que dans ces situations déconcertantes, nos corps sont serrés les uns contre les autres. Que chacun ait l'expérience ou non de la guerre, ce sont des moments de vives angoisses où les visages se figent avec dans la bouche ce mauvais goût d'incertitude. Lorsque nous nous relevons, une fois le danger éloigné, les liens réciproques se font plus intenses.

En bref, il existe une foule de raisons pour disqualifier un tel terrain, ou au moins pour le rendre impraticable. La dangerosité supposée du terrain n'est pas digne de discussion car elle ne conduit à aucune réflexion particulière sur le métier de sociologue. Ce qui est bien problématique, c'est le fait que le terrain soit fuyant, fluide et trouble. La seule manière de produire un semblant d'ordre, pour retrouver notre babitus de chercheur que le contexte singulier met en crise, c'est de collecter, de répertorier, de consigner, d'isoler les récits. Alors que la prudence, la raison et le confort sont mis à mal, l'écriture et la lucidité du questionnement de recherche sont les seules ressources pour faire oublier la situation quelque peu chaotique. D'ailleurs, les descriptions qui suivent font davantage ressentir plutôt que d'asséner des conclusions ou des qualifications hasardeuses sur le «monde social des combattants». En d'autres termes, l'essentiel réside dans le partage d'un questionnement, d'une expérience relativement peu commune dans le contexte de l'ethnographie. 


\section{Les ressorts subjectifs de la révolte : de la souffrance à la rage}

Il s'agit ici de trouver le ton commun qui existe en ce monde si particulier. Au cours de ces quatre voyages répartis en deux ans, bon nombre d'évolutions ont été repérées. Nous avons vu des hommes changer, s'endurcir, être saisis par la haine et la rage, jusqu'à sombrer parfois dans la crise et la déraison. Après avoir globalement qualifié ces dernières, nous décrirons les différents motifs évoqués par les combattants pour justifier leur engagement.

\section{Quand les motifs de l'action s'enrayent: de la détermination exaltée à la confusion}

Le 22 juillet 2012, notre premier voyage nous a conduit dans la ville d'A'zaz à quelques kilomètres au nord d'Alep. Celle-ci avait été libérée la veille de notre arrivée. Au poste-frontière, c'était l'effervescence de la victoire après une lutte opiniâtre. Ce 22 juillet, la ville était déserte. A'zaz était une ville privée de tout : sans lumière et dans le dénuement matériel. Si l'heure était à l'effervescence, il flottait sur toute la ville une odeur atroce et indéfinissable, tiède et tenace. Des ondes de détresse et de malheurs indicibles étaient diffusées par des milliers de tas de décombres et de traces humaines exposés au soleil brulant et impitoyable. On voyait partout sur le sol des tâches de sang, des automobiles et des tanks cabossés ou calcinés, des murs explosés.

C'est ici que nous passâmes l'essentiel de notre temps en compagnie d'Abou Kadour, Abou Rasheed, Abou Zaccharia et bien d'autres, tous combattants de l'ASL. En ce moment précis, tous se présentaient comme des « moudjahidines qui font la révolution ». C'est le terme qu'ils utilisaient et, à ce titre, il est irrécusable ${ }^{40}$. Le sentiment de participer au djihad existait mais était minoritaire. En ces moments, être «djihadiste » signifiait lutter pour le droit et la justice, valeurs qui se confondent avec la raison religieuse. La signification même du djihad n'était pas toujours partagée. Les disputes étaient nombreuses lorsqu'il fallait donner un sens précis à cette idée. L'importance de cette question dans les conversations ordinaires entre les combattants témoignait aussi de la place importante de la justification religieuse dès le départ du mouvement de protestation ${ }^{41}$. Les discours oscillaient entre un discours exclusivement religieux à la base même de la quête de justice et un discours plutôt humaniste qui visait la restauration de la paix et de la justice sans que cela ne réfère immédiatement à une transcendance religieuse. Plusieurs combattants nous certifiaient que le « djihad » était avant tout la défense pour la survie. Toute participation à la révolution, qu'elle quelle soit, participait au djihad. Le djihad n'est «pas seulement » la guerre offensive contre les mécréants. C'est ainsi qu'Abou Youssef, combattant, nous explique que «la

40. Laurens H., "Une révolution sans utopie (entretien) », Le Débat, 169, 2012, p. 18.

41. Pierret T., «Le Baas syrien face à l'islam sunnite », Le Débat, 168, 2012, p. 146. 
défense des églises fait partir du djihad. Tiens, par exemple, l'une des églises chrétiennes a été bombardée. Et bien défendre cette église, ça fait partie du djihad ». Pour ce dernier, le « moudjahidine » idéal est celui qui se défend contre l'occupation :

«Par exemple, les femmes sont des moudjahidines parce qu'elles préparent à manger aux combattants. Quand en France, il y a eu l'invasion allemande, les résistants français sont des moudjahidines. La différence, c'est qu'en 45, le monde s'est solidarisé. » (Extrait d'une conversation avec Abou Youssef)

Abou Youssef n'était pas un cas singulier. À cette époque, désormais révolue, le discours témoignait d'une ouverture réelle et s'attachait non seulement à déconfessionnaliser le conflit, mais aussi à contester la possibilité d'une radicalisation religieuse dans la société syrienne.

À ce moment, la préoccupation n'était pas d'ordre religieux. Les discours appartenaient au registre sémantique de la révolution ${ }^{42}$. Leur langage était d'une détermination et d'une régularité fascinantes. Il s'exprimait avec vigueur, résolution et exaltation. C'était le langage de la puissance et de la création. Les combattants nous expliquaient avec fierté combien la lutte les avait fait devenir d'autres hommes. Leurs mots laissaient entendre que leur emprise sur les choses s'étendait et se concrétisait. Leurs idées, leurs indignations passées, leurs colères longtemps réprouvées prenaient soudainement un droit et une existence réels. L'humiliation d'autrefois se trouvait tournée en son contraire. Dans leurs mots, on sentait qu'elle formait une volonté ferme à même de liquider les tentations de capitulation. Ils avaient la sensation de former des lendemains. Leurs discours laissaient apparaître qu'un nouveau monde s'ouvrait à eux ; un monde où le quotidien n'est plus fait d'accommodements à celui-ci, mais plutôt d'appropriation et d'actes concrets. Le monde sourd, destructeur, étouffant et oppressant semblait trouver sa propre chute. Il s'ouvrait un monde où les possibles étaient de nouveau exprimables et pensables. À les écouter, on sentait bien qu'il pouvait de nouveau jaillir en l'esprit le sens des possibles, comme en témoignent ces extraits d'entretiens ou de conversations entre les combattants.

«On est en train de construire la nouvelle Syrie. Bachar n’a pas le choix que de partir. Et il va partir bientôt, c'est sûr. On est proche de Damas. Demain, si Dieu le veut, on ira dans le centre [...] Je me vois dans quelques années, sur les collines surplombant Alep, contempler la ville tranquille, regarder les jolies filles et me réjouir de ces jours paisibles [...] Bachar est allé trop loin. Plus rien ne sera

42. La lecture de L'homme révolté d'Albert Camus (op. cit.) offre de nombreux mots pour qualifier le registre discursif révolutionnaire. Nous reprenons ici une partie des analyses de l'auteur pour qualifier le discours des moudjahidines. 
comme avant. Il a tué son peuple, mais son peuple est debout et ne s'arrêtera jamais. On a déjà goûté la liberté et on ne peut la sacrifier. [...] On mettra en place une démocratie où tout le monde vivra, où les institutions seront justes, où la corruption sera éradiquée, où on pourra vivre notre foi, où tous seront libres. »

Certes, Bachar El Assad et son système de gouvernement sont évidemment coriaces et ne se laissent pas aisément infléchir. Mais les combattants nous faisaient l'effet d'être habités par l'idée qu'ils pouvaient y parvenir. En juillet 2012, ils s'attendaient à une victoire imminente, sans doute avant la fin du ramadan. L'avenir se détendait et s'aérait. Dans leurs témoignages, on sentait bien qu'ils vivaient dans l'imagination, les rêves et le subjonctif. De toute évidence, leurs discours ne manquaient pas de sens du réel. Ils exprimaient d'ailleurs une volonté pressante de bâtir. La réalité n’était pas redoutée. Au contraire, elle éveillait les possibilités. La cellule armée se développait de manière considérable. Elle ne comptait plus les nouveaux déserteurs de l'armée régulière et les nouvelles recrues. En effet, chaque jour, l'enlisement dans le conflit et le retentissement considérable des exactions ou des morts de civils amenaient un grand nombre d'individus à solliciter leur admission au sein de l'ASL. En bref, les récits étaient ceux de l'engendrement. Ils ressentaient une délivrance, l'excitation, la fin de la peur, sans s'apercevoir de la sombre menace qui prit rapidement forme. Ces combattants nous frappèrent par leur étonnante facilité à espérer le changement de leur destinée au point que la lutte les entraînait dans son tourbillon.

Lors de notre second séjour en décembre 2012, puis surtout en avril et septembre 2014 dans la ville de Murek, la situation était tout autre. Ces hommes paraissaient dépassés par l'ampleur et la violence de leur soulèvement. Ils sont dorénavant entassés dans ces brigades et arrachés à la « normale quotidienneté ». C'est dans le creux des décombres qu'ils tentent de s'organiser une vie commune et un peu régulière tout en poursuivant le combat. Et c'est aussi, à mesure que le temps avance que ces hommes se font à cette nouvelle vie.

Murek est un coin reculé de la Syrie, pratiquement en son centre. Elle est entourée de collines et de champs de pistachiers, à quelques kilomètres de l'importante ville d'Hama et des premières terres alaouites. Murek est une petite ville qui ressemble plus à un bourg des environs d'Hama qu'à une ville. Elle est une ville de "front », presque entièrement détruite, un tas de ruines totalement anéanti. C’est dans l'une de ces rues dévastées que nous avons rencontré Abou Muhammad, Abou Omar, Abou Hassan, Abou Mossad, Abou Bakar et bien d'autres, combattants engagés dans une cellule liée au Front islamique. 
Indéniablement, la guerre transforme les hommes. Elle modifie la vie et fait advenir un nouveau « je $» 43$. Bon nombre de signes visibles manifestent le déclin de l'enthousiasme chevaleresque des débuts. Physiquement d'abord : la fatigue paraît saisir ces hommes et ces femmes. Elle se lit sur leurs visages cernés et agités. D'ailleurs, les combattants éprouvent du plaisir à nous faire deviner leur âge. En cœur, ils répondent : « ajoute cinq ans à mon âge réel et tu auras trouvé. »

Il n'est pas rare de les voir regarder dans le vide dans un miroitement confus comme s'ils ne voyaient qu'eux-mêmes. L'inquiétude semble tournoyer sans répit dans l'esprit des combattants. L'omniprésence de la destruction, de la mort, la montée de l'anarchie et des conflits entre cellules rivales, les bombardements permanents, l'habitude du carnage et des tremblements de terre, les nombreuses marches vers la mort sur les lignes de front, l'excitation nerveuse, les nuits sans sommeil et sans repos produisent un contexte sordide et terrible. Il fait grandir l'amertume en chacun d'eux. C'est un contexte apocalyptique mais réel.

Progressivement, ils deviennent moins troublés par ces faits quotidiens de leur nouvelle existence au point qu'ils semblent avoir oublié les jours d'autrefois. Ils s'habituent à des choses auxquelles ils n'auraient probablement jamais pensé s'habituer. La vie peut bien sûr y être tranquille et régulière. Mais ceci reste toujours provisoire. L'angoisse et l'inquiétude sont constantes sous l'air tranquille, insouciant et parfois nonchalant des combattants. Les temps annulés sont nombreux. Comme nous l'avons indiqué auparavant, le combattant se construit dans cette alternative entre la vie lente et l'inquiétude.

À mesure que le conflit s'enlisait, le sens des possibles se confondait au point que dès décembre 2012, et de manière plus significative en septembre 2014, il était rare d'entendre les combattants évoquer la victoire à venir, la Syrie à construire. Ils parlaient moins de l'avenir, comme si la cohérence d'ensemble se relâchait. En ces moments, le souvenir devenait tellement immobile; on ne rêvait plus. Les jours d'autrefois ne faisaient jamais l'objet de discussions entre les combattants. Même lorsque nous suscitions des échanges sur le passé, leurs paroles se brouillaient ou étaient d'une extrême concision. Chacun se détachait du passé. La lutte était en train de devenir " désespérée ", comme s'ils n'avaient plus rien à perdre. Ils étaient clairement las, brisés par l'enlisement d'un conflit qu'ils vivaient dans une parfaite solitude eu égard à la communauté internationale. Pourtant, la persévérance était au cœur du désespoir. Sans horizon victorieux clair, leur maintien dans le combat pouvait apparaître comme insensé. Mais étonnamment, ils continuaient jusqu'au bout, en étant presque certains de sacrifier leur vie. Dans leurs bouches, l'horizon était celui de la mort. Ils évoquaient leur mort à venir sur le ton de l'évidence :

43. Jünger E., La Guerre comme expérience intérieure, op. cit. 
«Notre situation est de mourir. Nos tombes sont déjà creusées. Elles nous attendent. On va tous y passer, personne n'échappera au trou. [...] Nous mourrons tous les uns après les autres. Nous n'avons aucune issue. C'est là notre destinée, c'est là notre combat pour Dieu. [...] Je vais mourir bientôt dans cette guerre. Au fond, il vaut mieux ça. » (Extraits de conversations avec deux combattants)

Concrètement, ils vivaient dans l'omniprésence de la mort. La mort, la préparation à la mort, était la toute dernière de leur justification. C’était le «dernier mot de la raison » disait Jünger ${ }^{44}$. Dans le désespoir, l'attachement à l'idée initiale rendait le combat irréversible. C'est, entre autres, ce qui conduisait le combattant, qui ne croyait pourtant plus vraiment à la victoire finale, à persister avec acharnement. Bien sûr, il y avait probablement aussi l'angoisse de la désagrégation du groupe de lutte, la perte de l'appartenance au groupe, alors que ce même groupe avait protégé l'individu des atteintes de la réalité extérieure ${ }^{45}$.

Cette crise de sens pourrait se résumer comme la distance croissante qu'il y a entre l'expérience sociale de la guerre et l'horizon d'attentes. L'avenir paraît lointain, indistinct, si bien que les individus ont le sentiment de n'avoir plus aucune prise sur le cours de l'histoire. C'est ce qui produit la rupture du désespoir. Ce désespoir se traduit concrètement dans leurs pratiques. La brutalité n'a presque aucune limite. Dorénavant, il y a quelque chose de bestial dans cette soif de vengeance. Le sang bouillonne et est omniprésent. L'empathie, y compris envers l'ennemi, paraît liquidée. Seul le désir d'anéantir fait loi. D'ailleurs, c'est au cours de nos deux derniers séjours que nous les avons vus acculés à quelques actes de désespoir ou que nous avons pu observer des situations « étranges »; ce genre de situation qui produit de l'embarras et une attitude un peu gauche. C'est ainsi qu'il leur est arrivé de manifester leur vengeance sur le corps des «prisonniers de guerre » en s'assurant de leur mort dans une rage folle, manifestant ainsi leur déraison ponctuelle. C'est l'enivrement de la guerre, favorisé par un contexte d'anéantissement, qui pousse chacun à la déraison. Et, tout au long de ces observations, il ne ressortait de ces combattants aucun signe de remords, de regret ou de repentir. La conscience paraissait sans danger, y compris après ces scènes de vengeance qu'ils avaient pu accomplir en certaines occasions. En d'autres termes, il s'installe comme un "sentiment d'évidence » au point que les hommes ne semblent plus douter de l'ordre des choses. Il est assez clair que ces hommes s'endurcissent; ils combattent et s'habituent à tuer tout en restant des hommes avec leurs virtualités de richesses spirituelles. C'est peut-être aussi ce que produit l'expérience sociale de la guerre. Cette expérience, tenace et éprouvante, ne renforce chez les combattants que la haine, la soif de vengeance et le fatalisme,

44. Jünger E., L'État universel suivi de La Mobilisation totale, op. cit., p. 86.

45. Revault d'Allonnes M., Ce que l'homme fait à l'homme. Essai sur le mal politique, Paris, Flammarion, 1995, p. 35. 
comme si leur engagement ne connaissait aucun retour. Et dans ces situations sordides et morbides, il n'est pas étonnant que certains se passent de toute mesure dans l'administration de la vengeance, comme s'ils agissaient sous l'empire du vertige ou du délire. Pour autant, ici ou là, il n'était pas rare d'attraper chez ces individus une souffrance intérieure, un regret de s'engager dans une guerre durable et sans horizon victorieux. Sur ce point d'ailleurs, plusieurs d'entre eux nous ont contactés après ces séjours pour tenter d'organiser leur départ en France.

Pour résumer, au départ, leurs explications quant aux motifs de leur engagement étaient claires et paraissaient parfaitement en ordre dans leurs têtes. Il était question de lutter contre l'oppression, la souffrance injustifiée, la torture, les humiliations quotidiennes, l'emprise d'un pouvoir tyrannique et la répression meurtrière. Ils exprimaient le fait que le seuil de l'intolérable était franchi. S'ils reconnaissaient qu'à la base ils n'étaient aucunement faits pour la guerre, ils nous expliquaient avec évidence que l'escalade rapide de la violence avait impulsé leur haine, seul ressort à même de justifier le combat armé. L'accumulation de la violence justifiait à elle-seule l'engagement armé en dépit de toutes les réserves morales que l'on pourrait éprouver en de pareilles circonstances. Six mois plus tard (décembre 2012), et encore davantage en 2014, leurs pensées paraissent plus confuses et plus éclatées comme s'ils apercevaient un immense espace incertain. C'est comme si l'on ne voyait que la forme d'un contour se dégager dans la confusion de mille courbes entrecroisées. Face à la confusion montante, il est tentant de naturaliser les motifs de l'engagement et de présenter son action comme une «nécessité historique ». La naturalisation des explications de leurs luttes est un processus « normal ». Le combat a besoin de détermination ; c'est ainsi que les choses devaient se passer, ainsi qu'ils devaient trouver la vérité et faire advenir la justice sans jamais lâcher la lutte vivante, complexe et ambiguë. Finalement, il est difficile de reconstituer les systèmes de raison des combattants. En effet, dans le quotidien armé, on réfléchit peu sur la lutte elle-même et sur la justification des valeurs. Le contexte intense de l'exaltation révolutionnaire tue les questions. Il n'est pas compatible avec un tel usage de la raison, avec un retour sur le fond des justifications qui sont nécessairement incertaines et hésitantes quand bien même elles s'appuient sur la conviction que la vie de misère et de malheurs excuse la violence. Il est difficile d'accueillir l'exigence réflexive en de pareilles circonstances. Il y a indéniablement quelque chose en eux qui se laisse aller à l'instinct. Il y a une certaine improvisation dans l'action dans un contexte d'effervescence collective qui emporte l'individu au point que, parfois, beaucoup nous donnent l'impression qu'ils font la guerre sans y penser, comme l'indiquent ces extraits de conversations avec des combattants :

« Je n’ai jamais hésité à m'engager. Très franchement, on pensait que ça allait durer quelques mois. Mais je peux te dire que je n'ai jamais hésité. Ma conscience est légère sur ce que je fais ici. [...] Il fallait 
utiliser les armes. Au début, on a une idée technique de ce qu'est une arme. Ce qui était compliqué, c'était d'attaquer nos cousins. Mais on avait pas le choix, il fallait bien se défendre. [...] Au début, on ne visait pas les soldats. Mais quand on a vu les réponses impressionnantes qu'ils nous infligeaient, on a dû les viser. »

Le paradoxe réside ici : d'un côté, il faut de la lucidité pour asseoir la force de la détermination, il faut évacuer les doutes et les scrupules, supprimer la critique désagrégeante. D’un autre côté, nous constatons l'impossibilité concrète d'une réflexion profonde sur le sens des possibles qui pourrait advenir. Ainsi, les combattants accomplissent des actes qu'ils méditent, réfléchissent et calculent tout en faisant droit aux mouvements les plus subjectifs appelant à la liberté élémentaire, à la dignité retrouvée, à l'excuse du malheur et de la détresse humaine qui fonde leur colère. Ordre et passion se confondent, émotion et froideur également.

Par ailleurs, lorsque nous les invitons à faire retour sur l'expérience du basculement subjectif qui les a conduit à la lutte armée, leurs explications sont loin d'être claires. Les entretiens témoignent assez clairement de la grande difficulté qu'ont les combattants à s'exprimer sur le caractère personnel de leur engagement. Cette difficulté à communiquer fait penser au travail de Hannah Arendt sur le procès d'Eichmann. C'est comme si les combattants s'étaient entourés de mécanismes de défense puissants contre les maux d'autrui et finalement contre la réalité elle-même. C’est comme s'ils se protégeaient des « atteintes de la réalité 46 ». Par exemple, dès lors qu'il faut remonter à l'expérience première de la violence, le discours passe toujours du « je » au «nous ». Autant les violences subies sont exprimées à la première personne du singulier, autant celles qui sont infligées à autrui sont figurées de manière impersonnelle. Ce n'est jamais une «volonté personnelle » qui expliquait l'administration de la violence, mais une nécessité collective, un « devoir impersonnel ». Généralement, dans le discours des combattants, l'acte individuel est immédiatement rapporté à un moment collectif de prise des armes. Il y a ici une identification élémentaire avec leurs « frères de lutte », ce qui est assez commun en ces circonstances 47 .

S’ils font référence aux situations humiliantes et désespérées qui étaient les leurs sous Bachar El Assad, les mots se font de plus en plus confus lorsqu'il s'agit de raconter le moment même du basculement. Chacun peut percevoir son propre basculement comme un chaos d'événements fortuits. Dans l'ensemble, tout paraît se mêler comme dans les tourbillons d'un torrent. L'esprit est confus, le cœur soulevé. Le basculement subjectif n'a pas de structures qui se donnent immédiatement à voir. On y trouve des relations causales telle-

46. Arendt H., Eichmann à Jérusalem : rapport sur la banalité du mal, Paris, Gallimard, 1966. 47. Browning C.-R., Des Hommes ordinaires, op. cit. 
ment nombreuses qu'elles restent inextricables. En tout cas, les expériences livrées dans ces récits ne sont pas toujours décrites par les combattants avec la même rigueur. Parfois, ils peinent à reconstituer certaines d'entre elles. Ils n'en retrouvent que des images isolées, parfois même sans rapport explicite entre elles, les unes étant toutes fraîches et décrites minutieusement, tandis que les autres sont recouvertes de poussière et se diluent dans le flou. Incontestablement, tout ce que les combattants nous disent n'est pas directement séparable de leurs revendications idéologiques, particulièrement exacerbées en temps de guerre. L'extrême détermination dont ils font preuve témoigne assez bien de ce contexte de guerre où le doute est liquidé et où il s'agit d'avoir prise sur les situations présentes dans ce qu'elles ont de plus critique.

\section{Le statut ambigu de la justification religieuse}

Depuis maintenant plusieurs années, la vie est constamment menacée et bloquée par la tragédie de la guerre. Aujourd'hui, contrairement au début de la guerre, l'avenir est dépourvu d'horizons victorieux. C'est donc l'avenir qui est hypothéqué. Face à la destruction, à la mise en ruine du pays, il n'est pas tout à fait étonnant de voir des individus s'en retourner auprès d'idées intemporelles, qui traversent les siècles et qui affirment la vitalité de leur propre société et de leurs valeurs. C'est ainsi que la religion a fait irruption dans les régimes de justification. D’ailleurs, comme le soulignait Albert Camus, l'engagement radical pour une cause incline à une lecture religieuse des phénomènes sociaux et politiques ${ }^{48}$.

Dès le début de la révolution, la religion a polarisé l'essentiel de l'attention des occidentaux, particulièrement sur la menace que pouvait faire peser la généralisation du motif conservateur dans la révolution syrienne. Cette question du rapport à l'islam est déroutante et globalement peu accessible aux commentateurs occidentaux. Il est difficile de le qualifier dans la mesure où, comme l'affirme Ellis Goldberg ${ }^{49}$, il est tout à la fois radical, rigoriste, extrême, tolérant et humaniste et extrêmement variable d'une cellule à une autre.

Dès les premiers jours des manifestations, les hommes se réunissaient dans les mosquées. La raison était plutôt sécuritaire car elles étaient, au moins au départ, relativement épargnées de la répression violente. C’est dans ces mosquées qu'ils écoutaient les cheikhs tels qu'Oussama Al Rita'i ou Krayyim Rajih 50. Des combattants racontent que c'est aussi en ces lieux qu'ils organisaient leur mouvement :

48. Camus A., L’homme révolté, op. cit.

49. Goldberg E., "Muslim Union Politics in Egypt: Two Cases”, in Burke E., Lapidus I. (eds.), Islam, Politics, and Social Movements, Londres, University of California Press, 1988, pp. 228243. 
«Au début, on se réunissait dans les moquées. Après le prêche, on s'organisait un peu. On tentait surtout de trouver les moyens de manifester sans qu'on se fasse arrêter. [...] Avant j'allais pas vraiment à la mosquée. Mais au début, c'est là où on se retrouvait pour bavarder. Les anciens nous parlaient. Et puis nous les jeunes, on était remontés. On croyait vraiment à ce qui se passait. Tout le monde était déterminé. On avait juste peur des policiers. Beaucoup d'entre nous ont été arrêtés. » (Extrait d'une conversation avec un combattant)

Au cours des manifestations auxquelles nous avons assisté (juillet 2012, A'zaz), les slogans religieux fleurissaient partout, se confondant avec les appels à la liberté comme on pourrait les trouver dans n'importe quel chant révolutionnaire. Par exemple, le 30 juillet 2012, nous assistions pour la seconde fois à une manifestation en « honneur des martyrs ». Il faisait nuit. Plusieurs centaines de personnes étaient là pour saluer les morts de la révolution. Comme à l'habitude, la manifestation était parfaitement organisée. Chacun se trouvait en ligne, les uns derrière les autres. Il y avait une espèce de mélange d'ordre et d'exultation révolutionnaire. Les chants retentissaient. Et les slogans étaient scandés avec toujours autant de ferveur :

«Votre silence nous tue, et autre qu'Allah nous n'avons pas de soutien. [...] Ô gouverneurs d'arabe, suffit du sabotage, la maison syrienne est combattue. [...] Ya Bachar El Assad, ne rêve pas de rester toujours. [...] Ya Allah accélère la victoire. » (Extraits de slogans criés lors de la manifestation à A'zaz en juillet 2012)

L’électricité fut brutalement coupée. Qu'importe, la manifestation continua avec le même enthousiasme dans l'obscurité, éclairée par les seuls phares des voitures qui l'encadraient. Les lumières des briquets, levés au ciel, forment comme un sillon d'étoiles d'espoir au milieu de la foule. Les chants se succédaient. Chacun d'eux mêlait religion et appel classique à la liberté et la justice :

«Al-cha'b yourîd i'dâm Bachar »: «Le peuple veut la peine capitale pour Bachar»

"ya ma ablâhâ el-houriyé »: "comme la liberté est belle »

"yasqout Bachar el-jabch»: "À bas l'âne Bachar»

"el-jaych el-hour, Allab yahmîh»: "L'armée libre, Dieu la protège »

"thawra thawra souriyé, souriyé, thawratna el-riz wa l-houriyé, bouriyé » : "Révolution, révolution, syrienne, syrienne, notre révolution est celle de la dignité et de la liberté, liberté. » (Extraits de slogans criés lors de la manifestation à A'zaz en juillet 2012)

50. Pierret T., « Le Baas syrien face à l'islam sunnite », art. cit., p. 153. 
Autre exemple, en décembre 2012, dès notre arrivée, nous étions invités à une «fête » organisée « en l'honneur de la révolution ». Celle-ci se déroulait dans la mosquée au centre ville d'A'zaz, rare bâtisse encore quelque peu préservée par les récents bombardements. Une centaine d'enfants étaient présents. La «fête »-c'est ainsi qu'on nous l'avait présentée - était organisée par la cellule de secours de la ville. Celle-ci était composée de combattants blessés qui n'étaient pas en état d'aller aux combats. Généralement, ils n’y restaient que le temps de leur rétablissement complet. La fonction de cette cellule n'était pas seulement d'organiser les distributions alimentaires et matérielles de première nécessité. Elle était aussi un lieu où les enfants pouvaient passer leur journée étant donné que l'école était fermée suite à sa totale destruction et à l'absence d'enseignants. Il s'y déroulait quelques activités pédagogiques : dessins, pièces de théâtre et enseignements religieux. À l'étage de la mosquée, une exposition de dessins d'enfants « sur le thème de la révolution » tenait place depuis quelques jours. Une trentaine de dessins étaient affichés représentant la douleur de la révolte, la fierté de l'insurrection et les rêves d'une Syrie libre.

Le jour de cette fête, les enfants étaient au centre. Telle une fête d'école de fin d'année, les enfants donnaient une représentation. Une dizaine de parents étaient présents. Quatre adultes organisaient la fête. L'organisation était ferme. Les adultes étaient omniprésents pour orchestrer la représentation, veillant à sa bonne tenue dans les moindres détails; ils faisaient taire les enfants quand ces derniers se laissent distraire, ils les présentaient, dirigeaient leurs chants, jouaient les chauffeurs de salle, s'occupaient de la technique, etc.

Cette fête constitue une mise en scène des principaux récits justifiant la révolution. En effet, les enfants furent conduits à interpréter les chants révolutionnaires les uns à la suite des autres. Ils jouèrent également quelques scénettes de théâtre reprenant des situations emblématiques de la révolution. Cidessous, un premier extrait de nos notes de terrain où l'on voit que les chants mêlent le religieux et le combat pour la liberté.

«Mon sang est arabe et syrien

À tous les visiteurs, bienvenue dans la Syrie libre [répété trois fois].

Mon sang est arabe. Je suis fier d'être syrien, je veux la liberté

Révolutionnaire

Syrie, on va te libérer

On va effacer la corruption

Il faut se départir de la peur qui est en nous

Assad n'est pas Dieu

Je n'ai pas besoin de toi

La liberté est notre eau

Bachar est contre Allah, il n'aime pas Allah. »

(Extrait des chants entonnés par les enfants) 
Extrait de notes de terrain : «Après ces quelques chants visiblement parfaitement connus de tous, interprétés avec application et ferveur, les enfants jouent une première scénette de théâtre. D’un côté, on trouve les manifestants qui revendiquent la chute de Bachar. Puis, en face, un autre groupe, en nombre moins important joue aux policiers. Les derniers fusillent les premiers. Les enfants portent alors en “martyr" l'un des leurs tué par balles. La scénette ne dure que quelques secondes. Une seconde scénette est organisée. Ici, il s'agit de jouer un homme qui cherche à prendre le pain devant quelqu'un d'autre. On lui explique qu'il ne dispose d'aucun privilège et qu'il doit attendre comme les autres. La scénette se conclut sur la phrase suivante : “Ce n'est pas parce que tu es de l’armée libre que tu as des privilèges”. »

Il n'est pas simple d'envisager concrètement le statut de l'islam dans les ressources subjectives à même de soutenir l'engagement des combattants. Il est clair que l'on peut observer une acculturation religieuse. Bon nombre de combattants nous ont confié avoir " découvert l'islam à travers la révolution [...] lire le Coran et en discuter librement [...] pratiquer les rituels sans avoir à craindre une quelconque répression [...] découvert Dieu à travers l'épreuve de la guerre » (extraits de conversations avec les combattants). Dès le départ, leurs discours dénonçaient la répression religieuse dont ils faisaient l'expérience : interdiction de prier, de jeûner, représailles, humiliations à cause de leurs pratiques religieuses, etc. C'est ainsi que l'idéalisation du combat a progressivement basculé vers des impératifs religieux qui lui étaient autrefois étrangers. La raison religieuse a progressivement recouvert la soif pure et simple de la justice.

Plus précisément, la manière qu'ont les combattants de thématiser l'islam dans leur engagement est des plus sommaire. Même s'il a été constaté une certaine contraction autour de la religion, la référence à l'islam est surtout utilisée dans le sens d'un projet général de moralisation des mœurs et de la vie politique. En attestent ces extraits de conversations avec des combattants du Front islamique :

«Je veux un État islamique, euh, un pays où on respecte l'islam, les valeurs de l'islam quoi. [...] Je veux une République islamique, quelque chose qui soit juste, qui plaise à Dieu [...].

Je veux un État qui se soumette à l'islam. On doit jamais voir des trucs comme la corruption ou la prostitution; il faut faire respecter nos valeurs. [...] Je veux des lois islamiques, que les institutions d'Allah soient appliquées. Je veux un État juste [...].

Je veux un État gouverné par l'islam. Je ne veux pas d'un régime qui conduise à des lois immorales parce que beaucoup le veulent. [...] 
Oui bien sûr que je veux un État islamique, je veux un État qui aide tout le monde. Je veux un pays sans injustice. »

(Extraits de conversations avec six combattants du Front islamique).

Comme ces citations l'indiquent, le projet politique révèle des aspirations à un moralisme islamique en institutionnalisant l'éthique de l'islam. Ce qui est frappant, c'est qu'en dépit de nos questions, aucun combattant ne discutait sur la stratégie de transformation de l'appareil étatique ou sur les formes d'organisation à même de faire vivre cet islam politique. Cela conduit à penser que le religieux constitue surtout une espèce de langage commun. Il fonctionne comme une formule à même d'asseoir la justesse de la cause révolutionnaire. La sémantique religieuse a l'avantage de refaire peuple là où ce dernier manque de plus en plus. L'islam est vécu comme une ressource à même de se sentir appartenir à un monde, en dépit de ce qu'il y a de plus en plus insupportable dans la situation actuelle. C'est le moyen le plus conséquent de susciter l'engagement dans la cause révolutionnaire sans briser l'ordre culturel. L'argument religieux constitue inévitablement une manière de susciter l'approbation quant au projet de transformation sociale qui est en train de se jouer. Car on comprend bien qu'une révolution se caractérise par la destitution des ordres anciens et dépossède les individus de leurs rapports ordinaires au monde. L'avenir du pays et de sa propre situation prend la figure de l'étrange et du menaçant. La banalisation de la violence, l'omniprésence de la destruction et de la mort produit la désolation. En ces circonstances de grandes désorientations, aucun monde n'apparaît clairement comme horizon.

Comme on peut l'imaginer, le flou général de la situation rend difficile la maîtrise du réel. Une des manières de le maîtriser consiste à lui appliquer un raisonnement épuré du contexte quotidien. Le recours au « djihad » est une opération radicale de réduction de la complexité du rapport de force en train de se jouer. En quelque sorte, la religion restitue aux combattants une certaine maîtrise du chaos dans lequel ils évoluent. Elle vitalise l'engagement au cœur même du désarroi. Comme Michaël Foessel 51 nous invite à le penser, cette expérience de l'effondrement est adoucie par le discours religieux qui refait ordre là où le monde apparaît brisé. Son omniprésence témoigne sans doute de cette aspiration à un ordre, à une certaine unité dans un contexte d'extrême dissociation. Albert Camus disait que dans l'anomie, on recherche des lois pour soutenir le sentiment de la justesse de la cause tout en simplifiant les causes et les visées de la lutte. C'est ainsi que l'islam ouvre des possibilités génériques et non singulières. Les points d'appuis à l'action sont généraux et généreux. Ici ou là, ils font référence au Coran, à la sharia ou à la sunna. Cependant, aucun principe n'en limite a priori l'étendue et aucune finalité n'en prescrit le déploiement. Le contenu même de la croyance semble peu importer. D'ailleurs, il est très rare d'entendre des discours déclinant de manière 
concrète comment l'islam pourrait trouver une traduction politique pratique. De ce point de vue, le discours religieux est éminemment général. Par exemple, rien n'est dit sur la volonté de Dieu en dehors du recours aux termes génériques de la «dignité » et de la « justice », tous deux renvoyant à la sacralité de l'être humain. Par exemple, Abou Youssef nous conduisait en voiture de la ville d'A'zaz à la ville d'Alep. Arrivés aux abords d'Alep, sur une colline environnante, nous nous sommes arrêtés le temps d'une courte pause pour attendre les instructions sécuritaires de circulation. Nous nous apprêtions à entrer dans la folie de cette ville. Nous la regardions. D’immenses colonnes de fumées grimpaient dans le ciel. À la vue de cette couleur grise et désolée, il nous a dit: "Voici ce que les hommes ont fait à l'islam ». Cette phrase a été énoncée dans un moment de spontanéité. Que voulait-il dire par cette phrase quelque peu brutale ? À notre sens, sa phrase ne portait pas un jugement strictement moral et religieux concernant la désobéissance des hommes. Il est à peu près certain que son énonciateur évoquait quelque chose d'autre qu'une simple vision moraliste de la société. Cette phrase exprimait la désolation et le caractère mortifère du paysage. Elle désignait la banalité du mal, la violence que les êtres humains sont capables de s'infliger mutuellement, l'autodestruction irréversible d'une ville autrefois rayonnante sur les plans culturel, artistique et intellectuel. Cette formule prend acte avec désolation d'un pays qui court à sa destruction. C'est cette capacité à s'abîmer, à s'enfoncer dans les tréfonds de la guerre que désignaient ces quelques mots énoncés avec dépit. Les mots d'Abou Youssef n'exprimaient sans doute aucune explication strictement religieuse du monde qui advenait devant nous.

De toute évidence, l'islam fonctionne ici comme une garantie contre l'errance. C'est un ordre général offrant la possibilité à une multitude de se rapporter à ce qui est hors d'eux-mêmes. Mais c'est une possibilité constante qui ne se laisse jamais prévoir concrètement. C'est une formule qui doit être comprise comme une expression de la finitude des existences singulières et non comme un terme objectif et partagé en fonction duquel il faudrait organiser le présent. Elle ne désigne aucune totalité close sur elle-même. L'islam est une sorte de cosmos de l'ordinaire à partir duquel l'individu se retrouve socialement. Le problème n'est certainement pas de fonder une norme d'action sur l'islam, mais d'examiner ce qu'elle implique pour une pensée du devenir politique et de ce qui menace l'existence des formes de vie minoritaires. Au fur et à mesure du temps, ces analyses deviennent sans doute obsolètes. Lors de notre dernier séjour, nous vîmes bon nombre de combattants rejoindre les rangs de l'État islamique ou du front Al Nosra. Ce n'est pas le motif religieux qui semble déterminant dans leur décision mais plutôt l'excellente réputation dont jouissent ces groupes. En témoigne ce combattant :

«Ce sont de vrais combattants, ils sont en première ligne, ils sont mieux équipés militairement, quand on combat pour eux on gagne plus d'argent (150 dollars par mois), si tu es un martyr, ils prennent 
soin de ta famille, et quand ils administrent des territoires, il n'y a plus de corruption et ils font régner l'ordre...»

En dehors de ces raisons pratiques, il faut reconnaître que l'enthousiasme pour ces groupes repose aussi sur le mécontentement et parfois la colère profonde qu'ils ressentent à l'encontre des Occidentaux qui, non seulement sont restés passifs mais, plus encore, interviennent avec une indifférence totale envers ce que les hommes de terrain pensent et veulent. En les écoutant, on voit bien que l'Occident paie ses longues années de mépris et d'humiliation. La religion est manifestement secondaire dans leur décision de rejoindre ces brigades. Pour eux, ces groupes, bien que parfois un peu « rigides », incarnent les valeurs d'islam, qu'ils voient avant tout, rappelons-le, comme un projet de moralisation des mœurs et du gouvernement des hommes.

Si les justifications religieuses trouvent dans le désarroi et la désolation un contexte favorable à leur épanouissement, il va de soi que ces explications se retrouvent également dans la présence éventuelle des soutiens institutionnels. Habituellement, ces derniers se constituent comme des «entrepreneurs de cause » au sens de Howard Becker 52 et tentent de confondre la religion avec le principe démocratique en réfléchissant aux voies possibles d'une « politique islamique ». Sur le terrain, ces entrepreneurs de cause se font rares. Le temps n'est pas encore celui de l'élaboration d'une théorie précise de l'islam politique. Dans les conversations entre les combattants, la grammaire politique est sommaire. Les principes sont généraux et généreux et ne font pas l'objet d'une traduction empirique.

\section{Le langage de l'indignation violente : l'excuse commune de la douleur conti- nue}

Dans la bouche des combattants, la religion n'est évidemment pas la seule ressource subjective à même d'asseoir la légitimité de la révolte. Elle est même secondaire par rapport au langage traditionnel de la souffrance et de la justice sociale. L'injustice, le déshonneur, la banalisation de l'indignité d'autrefois constituent le cour même des justifications de la nécessité de représailles, de la rage et de la fureur. Si ces hommes décident de renchérir sur l'effroyable misère de « leur » monde et acceptent de tuer, c'est parce que les limites du supportable étaient atteintes. La colère est fondée et est appelée à faire son propre droit ${ }^{53}$. C'est bien un discours de douleurs et d'indignation qui la justifie. C'est ce qui rend la révolte plus ou moins aveugle.

Il y a donc un fil conducteur au discours de la révolte. Celui-ci n'a pas

52. Becker H., Outsiders. Etudes de sociologie de la déviance, Paris, Métailié, 2012.

53. Sloterdijk P., Colère et temps, op. cit. 
significativement évolué d'un séjour à l'autre. Il se forme une « mémoire douloureuse » nourrie des souffrances et des humiliations passées. Le monde sous Bachar El Assad est montré dans sa déraison. Au départ de la révolution, la figure de Bachar El Assad était centrale. C'était l'ennemi à détruire. Construire un ennemi commun qui cristallise toutes les sources de souffrance individuelle fonde la capacité d'action d'individus isolés en un tout collectif. C'est une rationalité négative indexée sur Bachar El Assad. En quelque sorte et dans la bouche des combattants, il est l'incarnation hyperbolique du mal, son abcès de fixation.

Nous avons pu le voir en Tunisie, mais aussi en Égypte où le désormais célèbre mot d'ordre « dégage » rassemble à lui seul toutes les aspirations individuelles autour de la figure du «tyran ». C'est ainsi que les discours envers Bachar El Assad le condamnent sans appel. Et si parfois il est fait référence à l'influence de son entourage, ce n'est pas pour le dédouaner, mais au contraire pour inscrire la tyrannie de sa personne dans l'histoire du clan Assad.

Les combattants se pressent pour étaler le sentiment fondamental que Bachar El Assad et son système de gouvernement ont offensé leur vie. C'est lui qui aurait préparé les visages à la colère. L'oppression est décrite longuement comme pour démontrer que le seuil de l'intolérable est atteint. La violence est alors présentée comme le seul moyen de riposte. Les combattants se plaisent à rappeler qu'au départ, les manifestations étaient pacifistes. Si elles ont dégénéré, c'est en réaction à la répression aveugle et meurtrière de l'armée régulière. On nous explique que la misère, le sentiment "d'être enchaîné », « l'agonie devant la mort des civils innocents », le « gémissement devant la torture » sont autant d'actes qui ouvrent la voie à une radicalité violente, qui brisent la patience et le mûrissement des moyens pacifiques pour conduire un peuple à sa libération. Ces dénonciations radicales ont pour effet de reconfigurer le rapport au réel en justifiant la nécessaire fin de la résignation. C'est une façon de donner à la colère l'horizon de constituer l'impulsion décisive présidant à une réappropriation du monde.

\section{«Maman, ils ont détruit notre maison \\ Ils ont tué mon frère et mon voisin \\ Maman ils ont détruit notre maison \\ Ils ont tué mon frère et mon voisin \\ Mais ne t'en fais pas chère maman \\ Nous allons nous venger \\ Mais ne t'en fais pas chère maman}

Nous allons nous venger [5 fois]

Main dans la main, cartouche par cartouche, le peuple syrien est des milliards, nous protégeons ta terre chère Syrie, nous ne te tromperons jamais

Main dans la main, cartouche par cartouche, le peuple syrien est des 
milliards

Nous protégeons ta terre chère Rif de Hama

Nous ne te tromperons jamais

Nous ne disons que la vérité, chère maman, nous allons libérer notre maison

Maman ils ont détruit notre maison

Ils ont tué mon frère et mon voisin

Maman ils ont détruit notre maison

Ils ont tué mon frère et mon voisin. »

(Extrait d'une chanson interprétée par un enfant avec son père dans une mosquée près de Murek en septembre 2014)

Les discours de vie sont brûlants et d'une intensité incroyable. Ici ou là, l'exagération habille probablement les récits des combattants comme pour témoigner de l'intense souffrance vécue et la sensation d'étouffement qui sied à de telles circonstances. Au moyen de toute une série d'histoires singulières, qu'elles soient vécues par les combattants eux-mêmes ou par un de leurs proches, ils dénoncent la violence d'un pouvoir arbitraire. Le scandale n'est jamais loin et abstrait. Il est toujours incarné dans des existences singulières connues par le combattant et qu'ils relatent dans des conversations.

Les policiers sont venus dans mon magasin. Ils m’ont volé, puis ils m’ont emmené en prison. J'y suis resté quelques jours. Ils m’ont relâché moyennant une caution. [...] Dans une manifestation, mon frère a été tué. [...] J'ai passé plusieurs semaines en prison. Ils m'ont torturé et humilié. C'était un enfer. [...] Je travaillais à l'hôpital [...] quand j'ai vu l'armée détruire mon hôpital, j'ai su qu'ils étaient prêts à tout. J'ai constaté leurs violences. Les soldats ont tué trois patients civils sous mes yeux en affirmant que c'étaient des terroristes. [...] J'en étais à ma dernière journée de mon service militaire quand tout a commencé. J'ai vu des soldats tuer six personnes. Ce qui m'a choqué, c'est qu'ils étaient heureux de les avoir tués. »(Extraits de conversations avec trois combattants du Front islamique)

L'excuse commune à leur révolte est celle de la douleur commune engendrée par l'asservissement. Les combattants s'insurgent contre un pouvoir qui dégrade obstinément les hommes et leurs aspirations. La réinscription dans le monde se fait moins au nom d'un projet que d'une liberté à retrouver. Ils énumèrent l'intolérable du régime avec une aisance déconcertante, au point qu'il est même possible d'y repérer un répertoire limité de dénonciations. Les mêmes histoires sont racontées avec véhémence : répression religieuse, arrestations arbitraires, violences physiques et morales, enlèvements et disparitions, humiliations quotidiennes, injustices instituées, tortures, etc. Les récits sont poignants. Ils racontent la tyrannie et l'oppression. Ce sont des discours 
de l'insupportable qui construisent un « espace moral de l'intolérable 54 ». C'est comme s'ils exprimaient une fatigue de la vie, une lassitude à mourir dans des horizons fermés. Ils se présentent excédés ; ils n'ont plus à se forcer, à exiger d'eux-mêmes ce qu'ils ne peuvent "plus » faire, à se soumettre aux multiples humiliations dont ils faisaient autrefois l'expérience.

«On a été tués par les mains de nos propres frères, au nom de la sûreté nationale. Et pourquoi ? D'où venons-nous, nous ? Demandez à l'histoire, lisez notre page, nous avons été tués. » (Chanson de Samih Chakir).

La souffrance y est donc dénoncée sous une forme crue, de façon abrupte, sans circonlocution et atténuation, presque sans pudeur. La structure du langage suit de près le mouvement des émotions, qu'il s'agisse "d'exaspération » face à une situation étouffante, de "pleurs ", de «lamentations » ou d'une «voix éteinte » comme si elle était mise en branle par la brutalité de l'existence. Pour désigner le passé, le langage est tourné dans une négativité radicale au point que celle-ci impulse l'indignation morale. À A'zaz ou à Murek, partout où nous passons, les civils se pressent pour nous faire « constater » les désastres commis par l'armée régulière. Quand on ne nous montre pas les marques d'un corps mutilé, on nous fait visiter les hôpitaux, les écoles et autres bâtiments administratifs saccagés par les bombardements et criblés par les balles. On nous fait visiter des maisons familiales où des traces d'obus traversent à hauteur d'hommes les extrémités des murs. Là, on nous conduit au fond d'un jardin où sont entreposés les missiles et obus récupérés pour authentifier les preuves de l'intention meurtrière.

À l'occasion, de nombreuses images accompagnent ces discours. En guise de preuve, les combattants nous montrent des vidéos sur leurs téléphones. Ceux-ci sont peuplés de corps ensanglantés, démembrés, de peaux brûlées par les obus, d'enfants gisant à même le sol. On y voit aussi les vidéos et photos des martyrs, les images des combats dont ils se rappellent, des gens d'El Assad, des Iraniens ou des Libanais qu'ils ont capturés. On y voit des crimes, des exécutions, des corps mutilés, des visages morts, poussiéreux et hideux, les corps déchiquetés. Les combattants tiennent absolument à nous montrer « jusqu’au bout » ces images insoutenables comme pour faire preuve de la réalité de l'horreur humaine. Le spectacle de l'horreur et de la mort circule partout. Les images sont partagées, communiquées et regardées de manière quasi compulsive. Tout est fait pour s'habituer au carnage. C'est une forme de banalisation de la brutalité, une manière de la tolérer. Les combattants paraissent saturés d'horreur. Psychologiquement, cela prépare au spectacle de l'horreur.

54. Fassin D., «L'ordre moral du monde. Essai d'anthropologie de l'intolérable », in Bourdelais P., Fassin D. (eds.), Les Constructions de l'intolérable, Paris, La Découverte, 2005, pp. 17-50. 
Les frontières morales se détendent. Cette consommation compulsive de violence tend à favoriser des attitudes de destruction à l'égard d'autrui. C'est probablement ce qui contribue à ouvrir au relâchement de la distinction entre la cohérence et l'incohérence. D'ailleurs, nous avons vu une nette évolution entre 2012 et septembre 2014. En 2012, ils nous expliquaient qu'ils jugeaient les gens qu'ils capturaient au sein d'un tribunal. Ils ne le faisaient probablement pas toujours, mais ils avaient le souci de le prétendre. Aujourd'hui, les choses sont bien différentes. Il nous a été montré bon nombre de photos de combattants ennemis dont les têtes empilées formaient un tas de visages morts. C'est une sorte d'état de "désolation » ou plutôt de déréalisation du monde qui met en sommeil la conscience. Concrètement, cette sécession avec le monde se traduit par un oubli ou une impossibilité d'imaginer une vie sociale apaisée. Par exemple, au cours des entretiens, lorsque nous questionnions les combattants sur leur avenir personnel pour l'après-guerre, les mots leurs manquaient toujours. Cette difficulté à exprimer quoi que se soit est sans doute à relier à leur incapacité à le penser concrètement 55 .

De toute évidence, le registre de l'émotion est utilisé pour attester l'authenticité de la lutte. Les combattants usent également des négations successives pour donner une cause explicite à la radicalité de leur engagement. La radicalisation a besoin d'être naturalisée. Elle est l'effet d'une évidence. Face à "l'agonie », la violence est figurée comme le seul mode adéquat de la révolte. C'est ainsi que ces combattants ordinaires nous expliquaient leur passage à la lutte armée avec naturel :

« Nous n'avions pas le choix. C'était soit cela, soit nous mourions. L'armée de Bachar nous tuait lorsque nous manifestions. Les soldats tiraient à l'aveugle. Nous n'avions pas le choix. Il nous fallait prendre les armes. D’ailleurs, nous aurions dû les prendre bien plus tôt. [...] C'était normal de prendre les armes. Au début, on n'avait pas grand chose. On faisait des assauts brefs aux check points, mais c'était de la débrouille. [...] J'aime pas prendre les armes, mais voilà, que pouvait-on faire d'autres pour éviter d'être anéanti ? [...] Pour moi, c'était une obligation de s'armer. Chez moi, on avait un pistolet. J'avais une idée de comment l'utiliser. Il nous fallait nous défendre, c'est tout, trouver une solution pour se protéger. Alors, on s'est lancés dans des batailles de rue. Au départ, on shootait les check points. [...] La première fois, $j$ 'ai eu peur de tirer, mais on avait la force de combattre pour tous ceux qui étaient tués. Moi, j’ai pas hésité une seconde. » (Extraits de conversations avec quatre combattants du Front islamique)

55. Cette analyse a notamment été développée par Michaël Foessel dans : Foessel M. et Van Reeth A., La Méchanceté, Paris, Plon, 2014. 
Si les discours sur les motifs de la révolte sont autant unifiés, c'est aussi parce qu'ils sont propagés à travers des cellules de communication. Dans l'organigramme de la plupart des cellules de combattants, la communication a une place officielle. Elle joue un rôle non négligeable. Par exemple, à A'zaz en 2012, la cellule communication se limitait à trois personnes permanentes et quelques autres personnes par intermittence : ils étaient étudiants en géographie, en graphisme, en chimie, ou commerçants, transporteurs routiers, etc. C'est aussi ce que produisait le mouvement social à ses débuts : les révolutionnaires se formaient sur le tas. Ceux-là passaient de la condition la plus basse avant la révolution à l'honneur du pouvoir et à l'administration politique. Par exemple, c'est le cas d'Abou Kadour, le plus âgé des trois. Commerçant avant la révolution, il a été immédiatement affecté à la communication. Son rôle était de filmer les combats et de «faire tourner » les journalistes. Dans le bureau collectif des « communicants », une plaque en verre collectionnait les cartes de visite de la trentaine de journalistes internationaux qui s'étaient arrêtés à A'zaz. Fièrement et tel un trophée, il nous montrait les cartes de ceux du Monde, de CBS, de Fox News, d'Al Jazira et bien d'autres.

De petite taille, le sourire bien souvent en coin, Abou Kadour abordait son « nouveau métier » avec assurance. Son colt accroché à l'arrière du pantalon et ses lunettes de soleil lui donnaient un air fier. C'est avec le plus grand des professionnalismes qu'il montrait chaque décombre, narrait chaque combat. À l'époque, il avait répertorié toutes les victimes d'A'zaz. Chaque maison détruite, chaque famille touchée par les combats étaient soigneusement consignées. Les deux autres membres de la cellule communication étaient plus jeunes. Originaire d'A'zaz, ils avaient quitté la ville. L'un était étudiant en géographie à l'université de Damas. Il avait arrêté son master pour rejoindre l'ASL et filmait tous les événements de la révolution. Grand de taille, il était sur le front avec sa petite caméra de main. Il boitait un peu. Touché durant les combats, il offrait un regard déterminé : « dès que je serai remis de mes blessures, j'irai à Alep pour filmer les combats qui s'y déroulent, j'aimerais être aux côtés de mes frères. » L'autre, Abou Abdallah, était infographiste et il travaillait à Alep. Petit de taille, un bandeau accroché sur le bras, il affichait toujours un petit air moqueur et malicieux. Son allure était étonnante en temps de guerre. Elle rappelait davantage le petit minet tout droit sorti d'une boite de nuit, une image qui tranchait au milieu de cette zone de combat. Lui aussi avait tout quitté pour prendre en charge, à sa manière, la « libération du pays ». Un engagement qui n'empêchait pas les moqueries de la part des autres combattants qui les raillaient au motif de leur soudaine notoriété du fait que c'étaient eux qui « passent à la télé ». Ici, ils restaient les "petits » de la guerre, comparés aux combattants qui étaient quotidiennement au front et menaient les combats. "Ah oui les autres là, les "geeks", on les appelle les communicants, toujours avec leur Facebook et leurs ordis », nous disait d'un air moqueur un combattant. Ici, dans le bureau de la communication, on s'affairait : un seul ordinateur était en marche. Abou Abdallah gérait constamment les pages 
Facebook. Sur son ordinateur, il alternait à une vitesse incroyable sa navigation sur les pages Facebook, sur Youtube et sur Skype où il conversait avec quelques activistes. Sa journée était consacrée à récolter les informations émanant d'A'zaz ou d'Alep. Il gérait les nombreuses notifications de la page. Sur la page de la cellule de l'armée d'A'zaz, on comptait plus de 2000 personnes qui suivaient le fil d'actualités.

Il est évident que si le langage de l'indignation est fait d'une telle régularité, c'est aussi parce qu'il y a une organisation, une cellule de communication. La fonction de celle-ci est de rationaliser, régénérer et faire durer la colère.

\section{La fermeture paranoïaque : seul contre tous}

La situation géopolitique de la Syrie place les combattants dans un sentiment d'isolement face au monde. Il y a comme une « coupure paranoïaque ", ce qui est un processus classique en période de guerre ${ }^{56}$. Alors que Zine-ElAbidine Ben Ali et Hosni Moubarak ont été « dégagés » en un mois à peine, El Assad se maintient au pouvoir malgré la lutte menée depuis plus de trois ans maintenant. Ces hommes sont ainsi littéralement plongés dans un monde souterrain. Précipités dans une solitude concrète, ils vivent comme si les bruits du monde avaient décru jusqu'à s'effacer définitivement. Il y a comme une interruption du cours de la réalité. Le prolongement du conflit qui paraît sans fin n'est pas sans affecter l'unité des révolutionnaires qui doivent trouver des ressources à même de leur permettre de continuer à croire en la justesse de leur combat malgré l'enlisement qui engendre de nombreux morts et une situation humanitaire dramatique. Dès notre second séjour en décembre 2012, la situation que nous avions laissée quatre mois plus tôt nous avait paru changée. Nous retrouvions les combattants que nous avions quittés. Les retrouvailles furent heureuses, mais rapidement, la joie de se retrouver laissa place à la dureté de la situation sur place. Depuis si longtemps maintenant, ils étaient conduits à supporter l'attente d'une victoire qui se faisait de plus en plus incertaine et qui épuisait leur patience. L'enthousiasme chevaleresque se tassait. Les combattants, que nous avions connus éloquents et ardents, nous faisaient désormais l'effet d'être usés. S'ils continuaient à discourir sur le courage, la liberté et toutes sortes de vertus qui poussent à faire la révolution, leurs yeux n'en demeuraient pas moins flottants. La souffrance intense, l'espoir d'une victoire imminente et l'émotivité qui furent la leur étaient remplacés par le lexique de l'usure et de la fatigue. Trouver des ressources à son engagement dans un tel contexte passait pour eux par la création d'un discours de sublimation de leur lutte contre un ordre mondial injuste : «au début, nous menions une révolution contre un tyran. Aujourd'hui, nous sommes en guerre contre le monde qui soutient ce tyran », disait Abou Rashed, capitaine, tout juste sorti de dix jours de coma après avoir été touché au combat. 
L'engagement révolutionnaire s'épuisait manifestement sans s'éteindre. Une telle condition morale a eu des effets organisationnels. Les actions des révolutionnaires s'enchaînaient avec moins de fluidité, les coordinations s'enrayaient. L'orientation finalisée s'obscurcissait. Les conflits entre cellules se sont faits fréquents. Surtout, la construction pragmatique de la révolution, initialement centrée sur la destitution de Bachar El Assad, s'est transformée en un sentiment diffus d'irréalité où les revendications s'écrasaient dans les confusions. Parfois, les combattants prenaient le large par rapport à l'esprit révolutionnaire. Il leur arrivait d'errer dans des justifications qui manquaient parfois d'assises. Les motifs de l'engagement étaient moins clairs. Les combattants continuaient à condamner l'intolérable et l'oppression du régime, mais le combat se faisait de moins en moins au nom de l'idéal d'un monde meilleur. L'appel à un monde plus juste ne devint qu'un argument de circonstance. D’ailleurs, le nom de Bachar El Assad était beaucoup moins utilisé par les combattants alors que sa figure était centrale en juillet 2012. Il leur était de plus en plus difficile de se représenter un avenir du fait de leur impuissance à penser une issue. Le monde paraissait échapper à la plupart des anticipations, ce qui renforça encore une fois les certitudes religieuses.

Pour paraphraser Theodor Adorno ${ }^{57}$, «à la ruine objective de la vie matérielle, s'ajoute le désastre subjectif enfoui dans les profondeurs de l'individu ». La révolution a transformé l'existence en une suite de traumatismes dont la conséquence la plus importante est de faire oublier le passé, en particulier la quiétude routinière et les anciennes habitudes de vie en dépit des multiples oppressions qui étouffaient autrefois les aspirations. L'expérience du passé s'assèche, se vide, entraînant ainsi les hommes dans une conception fataliste de la lutte. C'est ainsi qu'un combattant nous a confié :

«Les révolutionnaires ont perdu le sourire. Ils ont oublié les jours heureux. La révolution est devenue la normalité. [...]. L'autre jour, mon fils de deux ans et demi regardait la demi-lune. L'air triste, il me regarda et me demanda : "Est-ce Bachar qui a tué la lune ?" [...] La guerre, mon ami, c'est tuer ou être tué. Alors, tu as intérêt à tuer. Notre destinée collective, c'est de mourir et je veux mourir pour l'islam. » (Extrait d'une conversation avec un combattant)

De toute évidence, le désespoir se lit sur ces visages cernés, vieillis et desséchés par l'épreuve radicale de la guerre. La guerre a des effets concrets. Ici, le sentiment de tourner en rond dans l'étroitesse des territoires libérés est vif. L'immobilisme de la lutte fatigue. Dans ces accès de fatigue, la conscience se relâche. Elle distrait les motifs de la révolte. La lutte parâtt être désormais dirigée «fatalement » vers l'impossible. 
Les combattants que nous avons rencontrés évoquaient avec dégoût l'idée de mourir dans l'indifférence brutale de la communauté internationale. C'est comme si leur vie était sans intérêt, géopolitiquement compliquée. Les combattants et les civils avaient la sensation d'être méprisés, oubliés, discrédités. Au détour d'un trajet en voiture, une personne nous criait : « saluez vos peuples, mais surtout pas vos gouvernements. » On nous posait également beaucoup de questions sur notre interprétation de l'inaction occidentale. On s'essayait maladroitement à quelques explications sans conviction. Pour eux, il était évident que les Occidentaux souhaitaient que la Syrie soit militairement entièrement détruite. Selon eux toujours, la peur de l'anarchie expliquerait également l'inaction car l'instabilité du pays le rendrait incontrôlable au niveau de ses frontières, particulièrement avec l'État d'Israël. Pour eux, il s'agissait d'affaiblir la Syrie pour la rendre impuissante sur le plan international. En outre, l'Occident a besoin d'institutions, qui, en l'état actuel des choses, avaient du mal à exister.

Il apparaît assez clairement que le dénigrement social des révolutionnaires musulmans dans la presse occidentale a généralement pour effet d'inculquer à ses minorités combattantes une image dévalorisée. Ce sentiment d'isolement, d'être «seul au monde ", de " combattre pour et contre le monde » est en creux de bon nombre de discours. Nous ne tentons pas de dire que ces allégations sont justes ou fausses, mais simplement de montrer à quels types de ressources les combattants ont recours pour donner sens à une situation qui s'enlise dramatiquement et qui, de fait, leur échappe en grande partie. Lorsqu'on incite les combattants à se justifier sur les motivations de leurs luttes, leurs discours sont configurés autour d'un rejet des stigmatisations médiatiques de la presse occidentale si bien qu'ils passent l'essentiel de leur temps à euphémiser les menaces inscrites en creux de certaines idéologies qui font leur nid dans le chaos ambiant. Le registre discursif de l'isolement est ainsi omniprésent. Cet enlisement dans la guerre et ce sentiment «d'être seul contre tous » agite l'esprit des combattants. Cette coupure paranoïaque est partiellement compensée par l'expérience du "vivre ensemble ", par ce contact entre les uns et les autres, lequel produit une sorte de décalage subjectif où l'individu se sent agi par une totalité sociale qui le dépasse. En effet, l'expérience de la guerre suggère une façon particulière de vivre ensemble. Les solidarités se radicalisent afin que chacun puisse prendre l'habitude de s'élever au dessus de lui-même pour affronter le combat. Dès le départ d'ailleurs, les récits des combattants expliquent que leurs peurs initiales ont été débordées voire transcendées par l'élan de leur mobilisation massive. Ainsi, à la déliaison générale, à l'extrême dissociation que suppose la guerre, à la fureur qui donne à la rage une forme concrète, il y a le "vivre ensemble », la consolidation d'une communauté de combattants "stigmatisés", comme en témoigne ce chant entonné par les combattants sur une ligne de front: 
«Mes chers amis,

Mes larmes sur mes joues avec l'amour du Prophète en moi,

Ils disent que je suis terroriste : je dis que j'en suis fier,

Notre terrorisme est aimé, appel de Dieu,

Notre terrorisme est aimé, appel de Dieu,

Où sont passés nos jours, quand nos cœurs étaient tranquilles ?

Ils sont partis en un clin d'œil, que c'est bon de s'en souvenir [deux fois],

Notre chef est Ben Laden,

Avec la puissance de la foi, nos larmes sont nos armes [deux fois],

Où sont passés nos jours, quand nos cœurs étaient tranquilles?

Ils sont partis en un clin d'œil, que c'est bon de s'en souvenir [deux fois]

Allah Akbar. ».

(Retranscription d'un chant entonné par tous les combattants à l'arrière, entre deux combats et à quelques mètres du front)

\section{Mourir en beauté : le corps érigé en symbole}

Au cours de nos quatre séjours, et particulièrement lors des deux derniers, nous avons assisté à beaucoup d'enterrements car il y a des morts tous les jours, et à chaque fois, c'est un mélange de grande tristesse, de lamentation et de rage. La figure $\mathrm{du}$ « martyr » est incontestablement centrale dans les ressources auxquelles les combattants mais aussi tout acteur de la révolution font appel pour donner sens à une mort omniprésente, à un quotidien où la vie a perdu son caractère sacré. Sur le plan des registres discursifs, le martyr est indéniablement un ressort de l'action politique. Alors que l'exaltation révolutionnaire s'infléchit, le discours prépare désormais à la mort. C’est la réalité politique qui tend à s'éclipser. Pour reprendre les termes de Myriam Revault d'Allonnes, « on est dans une sorte d'aveuglement héroïque (un héroïsme suicidaire) où l'activité s'est infléchie en passivité ». C'est comme s'il n'y avait d'autres solutions que de mourir. D'ailleurs, il y a une stylisation de la conduite à la mort ; une esthétisation qui d'achève dans « l'art de mourir [...] comme si la volonté de puissance se manifestait - au dernier moment - comme une libération de soi-même dans la mort 58 ».

Plus exactement, le martyr est un corps abimé et réduit à néant. Et c'est justement à travers la mise en scène des corps ensanglantés, blessés, que la figure du « martyr » prend tout sens. Hamit Bozarslan ${ }^{59}$ nous invite dans sa sociologie politique du Moyen-Orient à prendre acte du caractère éminemment politique de la figure du corps ${ }^{60}$. En citant Sartre, il rappelle que le pas-

58. Ibid., p. 71.

59. Bozarslan H., "Réflexions sur les configurations révolutionnaires tunisiennes et égyptiennes ", Mouvements, 66, 2011, pp. 11-21.

60. Bozarslan H., Sociologie politique du Moyen-Orient, Paris, La Découverte, 2011. 
sage à la violence est la conséquence d'une situation de "dos au mur », dans laquelle le corps-sujet n'a d'autre issue que le sursaut ${ }^{61}$. L'appel à l'acceptation du sacrifice de soi devient alors la seule issue pour des individus en quête de restauration de leur dignité. Dans ce contexte précis, et comme l'avait prédit Ali Shariati, philosophe et sociologue iranien, « le martyr entre en scène lorsque le moudjahid a échoué 62 ».

Les combattants encensent la possibilité de la mort. C'est une mort « virile ». Ils l'affrontent comme pour la rendre vaine. Elle devient moins improbable, moins abstraite et plus glorieuse car on lui trouve une cause noble. Glorifier la mort en construisant la figure du martyr revient à célébrer la rébellion qui persiste " héroïquement » dans la lutte, qui est, en quelque sorte, hors d'atteinte des structures de pouvoir qui s'abattent sur lui.

Glorifier la mort est aussi une manière de la transfigurer, voire de la rendre désirable. La mort est anticipée pour configurer la psychologie à une lutte opiniâtre. Elle est glorifiée comme s'il s'agissait de donner un but à la vie. Par la mort dans la lutte, on promet la possibilité de devenir un « grand » homme, un « héros » qui entrerait dans l'histoire du peuple de la Syrie libre par delà le temps. Il est clair que la mort est exaltée. Elle vitalise encore davantage la haine et l'ardeur de la vengeance. D'ailleurs, il y a de quoi glorifier la mort. En temps de guerre, cette idée d'avoir combattu et persévéré jusqu'à la mort rend digne. C'est la haute idée, bien tenace, d'exposer son existence non pour elle-même, mais pour l'idée. C'est ce que Jünger appelle la «bravoure 63 ». Celle-ci était encore tenue comme une qualité remarquable dans le rang des «insurgés ». C’est ainsi que la mort est ennoblie et rendue héroïque.

En Syrie, la mort est célébrée comme l'exemple typique de la dignité du combat, contre l'avilissement de l'existence, contre la douleur de l'agonie dans un contact où, finalement, «il y a bien plus à craindre que la mort 64 ». La vie importe soudainement moins. Elle est subordonnée au désir de justice. La guerre se motive aussi ainsi. La détermination au combat s'appuie sur l'idée qu'il vaut mieux mourir que survivre à l'indignité d'un soulèvement avorté. Ce sentiment de « devoir aller au bout » et de témoigner d'une relative indifférence à la vie pour le devenir de leur cause est évidemment une des modalités classiques de l'exaltation révolutionnaire et guerrière ${ }^{65}$. Leur existence ne leur appartenait plus. L'idée l'emporte sur le tout. Les combattants sont habités tout entier par l'idée que c'est le «devenir qui est au dessus de la vie ». C'est ainsi que dans un premier temps, les combattants nous semblaient être comme

61. Idem.

62. Khosrokhavar F., Les Nouveaux martyrs d'Allah, Paris, Flammarion, 2002.

63. Jünger E., L'État universel suivi de La Mobilisation totale, op. cit., p. 87.

64. Adorno T. W., Minima Moralia, op. cit.

65. Duclos L.-J., « Le quasi-contrat du combat suicidaire », Cultures $E$ Conflits, 63, 2006, pp. 2546. 
des « icônes » aux yeux des habitants des territoires libérés. Par exemple dans la rue, les jeux d'enfants sont effrayants; ils se plaisent à jouer aux « martyrs » s'effondrant à simuler une mort au combat, pendant que les autres camarades prennent l'allure qui est de mise en ces circonstances. En observant la théâtralité parfaite avec laquelle ils jouaient ces scènes, on comprend aisément que le « corps martyr » devient créateur d'une symbolique de sens : celle de la lutte pour la dignité, car il est assez frappant de voir tous ces enfants connaittre absolument par cœur les moindres détails de la cérémonie encadrant les funérailles que l'on offre aux martyrs. Le martyr n'est pas lavé, il est enterré avec les traces de sa lutte, sans linceul, ses habits et ses blessures attestent de son sacrifice. Quand un martyr tombe à A'zaz ou à Murek, ce sont tous les hommes présents qui descendent dans la rue, Kalachnikovs braquées vers le ciel, et qui suivent le corps porté au cimetière. Les chants religieux dans ce contexte font l'effet de slogans appelant à la lutte et les prières sont pleines de rages et non de tristesse. D'ailleurs, il est d'usage de «féliciter » les membres de la famille endeuillée en guise de condoléance. Le deuil d'un martyr renouvelle la foi des engagés dans la lutte et crée la colère nécessaire à ceux qui n'avaient pas encore pris les armes. Au début d'ailleurs, il n'était pas rare de voir après chaque mort, des enrôlements de membres de la famille ou de voisins du martyr dans la rébellion. Il est indéniable que la figure du martyr crée de l'engagement. La pression de la mort soude.

À Kafer Zita, nous avons assisté à l'enterrement de deux combattants de la cellule Jabhat Nosra. Au moment où les deux hommes sont mis en terre, nous écoutions le chef de la brigade sermonner la masse des combattants agglutinés autour des deux tombes :

"Ceci est notre situation, la situation de tout le monde, tout le monde ici a un trou, un trou qui lui sera creusé de cette façon, donc soyez prêt. Un savant a dit : "pleure pour toi-même avant qu'on ne pleure pour toi”, et maintenant un de nos frères pleure pour Abou Zayd, et moi je te dis pleure pour toi-même, et sois prêt pour cet instant, je vous jure que nous allons tous passer par ce moment, aucun de nous n'échappera à ce trou.

Donc demandez à Dieu : "permettez-moi d'être parmi ceux qui sont prêts à être dans ce trou", prenez soin de vous et arrangez-vous, regarde ton état mon frère musulman, regarde ton état, es-tu prêt à te tenir devant Dieu dans cet état comme tu l'es maintenant ? Es-tu prêt à te tenir devant Dieu en ce moment ? Dieu est grand ! La mort ne connaît pas un lieu précis, ni une cause précise ni une heure précise et ni un âge précis, elle vient soudainement, vous ne la sentez même pas. La mort est un droit pour nous, c'est ce que tu essayes de dévier, ce qui veut dire que c'est ce dont tu as peur, ou ce que tu fuis. Notre frère vient de nous quitter, il nous a quittés pour aller vers la 
vraie vie. Est-ce que tu es prêt pour ce moment mon frère ? Sois prêt à te tenir entre les mains de Dieu, prends soin de toi, prends soin de toi ! Est-ce que tu abrèges tes prières, ton djihad, ton travail ou ce que Dieu t'a ordonné de faire ? Si c'est le cas, confesse-toi et repenstoi tous les jours afin que Dieu te pardonne, et qu'il fasse en sorte qu'il t'emporte qu'après avoir dit “Je témoigne qu'il n'y a d'autre Dieu qu'Allah et je témoigne que Muhammad est le Messager de Dieu”, demande à Dieu qu'avant ta mort tu puisses dire “Je témoigne qu'il n'y autre Dieu qu'Allah et je témoigne que Muhammad est le Messager de Dieu” travaillez mes frères, la mort est un droit qui vous viendra, donc faites-le pour l'amour de Dieu, faites-le pour l'amour de Dieu, faites-le pour l'amour de dieu ! Demandez à Dieu d'accueillir notre frère, je demande à Dieu de t'accueillir Abou Zayd! Soyez sûrs et certains que personne d'entre vous ne fuira ce trou, je vous jure que vous allez tous y passer, vous allez tous être dans ce trou, alors arrangez-vous et pleurez pour vous-même avant qu'on ne pleure pour vous ! Tous à voix haute "Dieu est grand, Dieu est grand, Dieu est grand". "

(Retranscription du discours accompagnant l'ensevelissement de deux combattants du front Nosra)

Ce discours, énoncé au moment où deux « martyrs » étaient ensevelis, témoigne assez bien du caractère « interchangeable » des hommes. Il illustre clairement l'assèchement de la signification donnée à la mort. Mourir devient « sinistrement un mode de vie 66 ».

Dans le bureau central du QG d'A'zaz, les photos des martyrs étaient affichées sur le mur. En décembre 2012, il y avait déjà tellement de morts que la place manquait pour les honorer et les garder en souvenir. Les morts étaient entassés. Le mur était étouffé de ces visages. Car là était bien le problème : la mort se banalisait. Pire, elle se rationalisait. Par exemple, à A'zaz comme à Murek, les tombes étaient creusées avant même qu'elles soient habitées. D'ailleurs, dans les cimetières, on reconnaissait l'odeur lourde et tenace de la putréfaction. «C'est comme si on manquait de respect à la mort » soupira Abou Kadour, un combattant de l'ASL. C'est ainsi que la possibilité de devenir un héros devint dérisoire. Ni gloire ni renommée n'étaient maintenant espérées. En conséquence, l'engagement dans la lutte devint plus fataliste et plus déraisonnable. Dès décembre 2012, on observait un resserrement des justifications sur la possibilité de la mort autour d'une idée épurée : il faut connaître le malheur de la fosse, la profondeur tragique du corps en danger pour entrevoir la possibilité de l'engagement total dans la lutte, pour espérer assister à l'aurore difficile d'un monde rayonnant. L'espoir de devenir un mar-

66. Revault d'Allonnes M., Ce que l'homme fait à l'homme. Essai sur le mal politique, op. cit., p. 29. 
tyr et un héros n'eut certainement pas manqué. Mais, au moment où les morts s'accumulaient, la mort se fondait dans une transparence impersonnelle. C'est un mort sans nom. Le combattant ne devient qu'un « presque personne ». La mort devient brutalité et inconsistance. En quelques sortes et pour reprendre les mots d'Ernst Kantorowicz, les vies humaines en finissent par « ne plus être sacrifiées mais liquidées 67 ».

\section{Conclusion}

Cette étude ethnographique permet d'avancer plusieurs conclusions. Premièrement, il s'agissait de montrer les difficultés constitutives à la démarche ethnographique en un tel contexte. L'écriture ethnographique appelle à une mise en ordre et en sens. Elle est une manière d'objectiver et de fixer les raisonnements ordinaires des combattants en ces circonstances tout à fait particulières. Seulement, dans un contexte chaotique, il est périlleux de figurer un « ordre des choses » tant celui-ci paraît précaire et incertain. De la configuration physique du paysage aux motifs instables de l'engagement dans la lutte armée, jusqu'à la confusion liée à la multiplicité des causes de l'engagement, l'ethnographe est tenu à un travail quasiment impraticable de rendre compte dans toute sa rigueur scientifique du sens des conduites des combattants. C'est la raison pour laquelle, dans ce texte, le langage ethnographique a quelque chose de relâché dans le but de faire sentir plutôt que de faire connầtre. Le compte rendu veut maintenir cet état d'incertitude et de précarité pour faire droit à l'instabilité générale des rationalités ordinaires et des formes d'existence spécifique aux combattants.

En dépit de ces considérations méthodologiques, et c'est là le second temps de l'analyse, les différents récits des combattants présentent une cohérence étonnante. Il existe un répertoire commun des dénonciations. Leurs récits laissent clairement apparaître que les « événements critiques » liés aux répressions violentes du pouvoir à l'encontre des manifestants pacifistes ont accéléré le vent de la révolte. Rapidement, ces événements critiques ont été articulés avec des expériences passées de l'injustice au quotidien. Les combattants témoignent avec ardeur des multiples souffrances, humiliations et injustices dont ils ont depuis longtemps fait l'expérience. Ces dernières sont systématiquement incarnées dans des histoires singulières proches du combattant, ce qui facilite indéniablement l'identification empathique. La montée en radicalité de l'engagement politique se constitue dans l'émotion et ne se soumet à la direction de l'intellect qu'en seconde instance. L'émotion est cette force qui contribue à la mise en mouvements toujours collective.

Troisièmement, il est apparu assez clairement que les motifs du soulèvement évoluent et se traduisent par une transformation du lexique utilisé par 
les combattants. Au départ, les récits transpiraient la détermination, l'imagination, les rêves et le subjonctif. Le sens des possibles trouvait un contexte favorable à son épanouissement. La colère était toute droite dirigée contre Bachar El Assad, figuré comme un tyran et responsable de la violence meurtrière des « révolutionnaires ». Mais à mesure que les hommes s'enlisent dans le conflit, que les espoirs se tassent, que les lendemains ouvrent à un immense espace incertain, les ressorts subjectifs de la lutte armée se relâchent. Le lexique de l'usure et de la fatigue se substitue à l'exaltation révolutionnaire. L'extraordinaire se banalise. Pour le dire autrement, au commencement, les combattants se dotent d'un passé et d'un futur homogènes. Il existe une constellation de souvenirs qui supportent la lutte. Le souvenir de la misère, localisé et individualisé, est entretenu et rationalisé pour fonder les prémisses d'une unité possible. Cependant, quand le présent se ferme, les passés et les futurs communs se contractent voire s'effondrent. À mesure que l'immense champ initial des possibles se rétrécit, le champ lexical du passé et de l'avenir disparaît. La lutte tend à être dépotentialisée. Seule la présence au présent, la vie au jour le jour, réactualise la lutte armée. C'est ainsi que l'idéalisation du combat a progressivement basculé vers des impératifs religieux qui lui étaient autrefois étrangers. La raison religieuse s'est rigidifiée et a progressivement recouvert la soif pure et simple de la justice.

Enfin, le rapport à la célébration de la mort est tout à fait symptomatique des évolutions mentionnées précédemment. Si au départ celle-ci est encensée et centrale dans les ordres de grandeur qui construisent la dignité du combattant, sa valeur sociale s'amoindrit à mesure que les morts s'accumulent. La mort se fond dans une transparence impersonnelle. Elle devient banalité, brutalité et inconsistance. C'est là le degré ultime de l'altération des ressorts subjectifs qui conduisent à la lutte armée, ouvrant alors la voie à une naturalisation totale de l'engagement, lequel devient motivé en grande partie par le caractère irréversible des efforts préalablement engagés. 